\title{
Coherent Manipulations in Semiconductor Nanostructures
}

\author{
T. Amand, X. Marie, P. Renucci and E. Vanelle \\ Laboratoire de Physique de la Matière Condensée, UMR CNRS 5830 \\ INSA, 135 Avenue de Rangueil, 31077 Toulouse cedex 4, France
}

This contribution presents recent results on spin manipulation by optical pulses in various semiconductor nanostructures such as quantum wells, microcavities, quantum dots. The potentialities of temporal coherent control, as well as spin dynamics under magnetic field are investigated, using the current ultrafast emission spectroscopy techniques.

PACS numbers: 42.50.Md, 71.35.Ji, 71.36.+c, 78.47.+p, 78.67.De, 78.67.Hc

\section{Introduction}

Coherent manipulations in semiconductor nanostructures have been the subject of an intense scientific activity for a few years, particularly in relation with potential applications such as quantum information processing. In the latter, information encoding is generally designed by achieving coherent linear superpositions of quantum states in two- (or eventually more) level systems, which are generally denoted as quantum bits ("q-bits"). The first attempts in these directions were made in atomic physics [1], where, however, the possibility of integrating a sufficiently high number of "q-bits" seems questionable. This difficulty could be overcome in solid state devices. On the other hand, the problem of decoherence is much more critical in condensed matter.

In this paper, we will review different spin manipulations in semiconductor nanostructures (quantum wells, quantum dots). Typically, two kinds of limiting processes can be identified with respect to this aim: optical coherence relaxation, conveniently characterized by the optical dephasing time $T_{2}$, and spin coherences relaxation, characterized by longitudinal (transverse) spin relaxation times $T_{s 1(2)}$. In the case of quantum well excitons, for instance, the optically active doublet ( $J=1, J$ - exciton angular momentum) can be viewed as an effective spin. After recalling the basics of optical pumping in relation with exciton fine structure 
(Sec. 2), we will show that it is possible to create an arbitrary coherent superposition of these spin states by using a sequence of phase locked optical pulses with orthogonal polarizations, using a temporal coherent control scheme (Sec. 3). Such experiments can be extended to the case of microcavities in the strong coupling regime, where spin dephasing times can be increased to some extent. Spin manipulations by optical pulses is indeed limited here by the short exciton (or polariton) optical dephasing times. With this respect, quantum dots, where quantum confinement should strongly reduce dephasing processes, are good candidates: at low temperature, we show that the spin coherence decay time is much longer than the electron-hole pair lifetime (Sec. 5). However, the problem of sample inhomogeneity should be overcome, by addressing single boxes.

Finally, experiments designed to manipulate spins and their coherences under magnetic field will be presented (Sec. 4). In particular we will stress on exciton spin quantum beats in quantum wells in the case of correlated or uncorrelated electron and hole spins, and conversion between optical spin orientation and alignment in quantum boxes (Sec. 5).

\section{Optical pumping experiments and exciton fine structure in semiconductors nanostructures}

The understanding of optical pumping experiments performed on excitons in semiconductors lie on the knowledge of their fine structure. For excitons in quantum wells (QWs), e.g. in the typical GaAs/AlGaAs structures grown in the [001] direction, the combined action of the confinement, which quantizes the states along the growth axis $O z$ and the spin-orbit interaction simplifies the formulation of the quantum states with respect to the bulk situation. Practically, the symmetry of the system is $D_{2 d}$ and we can consider that the lower exciton states are obtained by the binding of an electron with a spin $s_{z}= \pm 1 / 2$ and heavy hole with an angular momentum projection $j_{z}= \pm 3 / 2$. These excitons, the so-called heavy-hole excitons and labeled $X H$, are located at about $10 \mathrm{meV}$ below the gap for narrow quantum wells of type I with a typical width of $3 \mathrm{~nm}$. The $X H$ states are described in the basis of the exciton angular momentum $\widehat{\boldsymbol{J}}=\widehat{\boldsymbol{s}}+\hat{\boldsymbol{j}}$ ( $\widehat{s}$ and $\hat{j}$ are the conduction electron and hole angular momentum operators, respectively), which is diagonal with respect to the spin-orbit interaction: $\left\{\left|J_{z}\right\rangle=\left|j_{z}+s_{z}\right\rangle \equiv\left|j_{z}, s_{z}\right\rangle, j_{z}= \pm 3 / 2, s_{z}= \pm 1 / 2\right\}$. The intra-exciton electronhole exchange splits the degeneracy of the $X H$ quadruplet. Practically, two contributions are distinguished [2]:

(i) the short range one, which can be written with the effective Hamiltonian

$$
H_{\mathrm{sr}}=\delta_{\mathbb{K}, K^{\prime}} \sum_{i=x, y, z}\left(a_{i} \hat{j}_{i} \widehat{s}_{i}+b_{i} \widehat{j}_{i}^{3} \widehat{s}_{i}\right),
$$

where $\widehat{s}_{i}$ and $\widehat{j}_{i}$ are the components of the electron spin and hole angular momentum operators, respectively, and $a_{i}$ and $b_{i}$ are the coefficients determined by 
the exciton radial and center of mass motion (in $D_{2 d}$ symmetry $a_{x}=a_{y}$ and $\left.b_{x}=b_{y}\right)$ and $\boldsymbol{K}$ is the exciton center of mass wave vector. $H_{\mathrm{sr}}$ splits the optically active doublet $\left\{\left|J_{z}\right\rangle=| \pm 1\rangle \equiv| \pm 3 / 2, \mp 1 / 2\rangle\right\}$ from the quasi-degenerated pair $\left\{\left|J_{z}\right\rangle=| \pm 2\rangle \equiv| \pm 3 / 2, \pm 1 / 2\rangle\right\}$ with a quantity $\delta_{X}$ of about $0.1 \mathrm{meV}[3]$;

(ii) the non-analytical or long-range one, which can be described for $X H-1 S$ $(J=1)$ exciton states by the following effective Hamiltonian:

$$
H_{\mathrm{lr}}=\hbar \Omega(K)\left[\frac{\left(\widehat{J}_{x} K_{x}+\widehat{J}_{y} K_{y}\right)^{2}}{K^{2}}-1\right] \delta_{\boldsymbol{K}^{\prime}, \boldsymbol{K}^{\prime}},
$$

where $\Omega$ is a linear function of $K$ at small $K$ values $[2,4] . H_{\text {lr }}$ splits the optically active doublet, and can be identified to the longitudinal-transverse splitting for the exciton modes with non zero linear momentum [5]. It does not couple the $X H(J=2)$ states. As the exciton binding energy, the exchange interaction is enhanced by the confinement in the QW structure, the exchange terms being proportional to the square of overlap integral between the electron and the hole wave functions. As a fact, the exchange energy $\delta$ is maximum for $1 S$ states, and decreases rapidly for excited bound states (the order is about $0.04 \delta$ for $2 S$ states) or unbound states [6]. The optical selection rules in dipolar approximation are such that the states $\left|J_{z}\right\rangle=| \pm 1\rangle$ are coupled to the optical mode $\sigma^{ \pm}$ of the same angular momentum. The $\left|J_{z}\right\rangle=| \pm 2\rangle$ states, which cannot be excited with one-photon transitions, are called "dark states". In an optical pumping experiment using a short resonant $\sigma^{+}$polarized optical pulse, the $|+1\rangle$ exciton population will relax towards thermal equilibrium between the $|+1\rangle$ and the $|-1\rangle$ states, due to the longitudinal-transverse splitting and collisions experienced by excitons as described below. The circular polarization degree can be evaluated in the frame of the exciton density matrix formalism. Using the isomorphism defined by $\{|+1\rangle,|-1\rangle\} \rightarrow\left\{|+1 / 2\rangle^{X},|-1 / 2\rangle^{X}\right\}$, it is convenient to define the exciton effective spin $\widehat{\boldsymbol{S}}^{X}=\frac{\hbar}{2} \boldsymbol{\sigma}, \boldsymbol{\sigma}=\left(\sigma_{x}, \sigma_{y}, \sigma_{z}\right)$ representing the Pauli matrices acting on the $X H$ optically active subspace. Introducing the exciton density operator $\widehat{\rho}$, the mean exciton spin is then given by $\boldsymbol{S}^{X}=\operatorname{Tr}\left(\hat{\rho} \widehat{\boldsymbol{S}}^{X}\right)$. The circular or linear polarization degrees of excitons are then simply given by

$$
\begin{aligned}
& P_{\text {circ }}=2 S_{z}^{X}=\frac{\rho_{1,1}-\rho_{-1,-1}}{\rho_{1,1}+\rho_{-1,-1}}, \quad P_{\text {lin }}=2 S_{x}^{X}=\frac{\rho_{-1,1}+\rho_{1,-1}}{\rho_{1,1}+\rho_{-1,-1}}, \\
& P_{\text {lin }}^{\prime}=2 S_{y}^{X}=\frac{\rho_{-1,1}-\rho_{1,-1}}{\mathrm{i}\left(\rho_{1,1}+\rho_{-1,-1}\right)},
\end{aligned}
$$

the two linear polarizations being taken along the $(O x, O y)$ and $\left(O x^{\prime}, O y^{\prime}\right)$ reference axis (the latter is rotated by $\pi / 4$ around $O z$ from the former). Finally, the exciton polarization dynamics can be traced out by measuring the optical polarization characteristics of the time resolved secondary emission normal to the sample surface. For instance 


$$
P_{\text {circ }}(t)=\frac{I^{+}-I^{-}}{I^{+}+I^{-}}, \quad P_{\text {lin }}(t)=\frac{I^{x}-I^{y}}{I^{x}+I^{y}},
$$

where $I^{+}$and $I^{-}$are the intensities of right and left circular components, and $I^{x}$ and $I^{y}$ are the linear ones along $O x$ and $O y$. For instance, Fig. 1a displays $P_{\text {circ }}(t)$ after the resonant excitation of $X H$ by a $\sigma^{+}$pulse. The characteristic relaxation time, the so-called longitudinal spin relaxation time, denoted $T_{s 1}$, is of the order of a few tens of picosecond at low temperature [7]. It is not sensitive to the exciton density, at low or moderate densities $\left(<10^{10} \mathrm{~cm}^{-2}\right)$. It decreases when the confinement increases as predicted by the theory developed by Maialle et al. [4]. In addition, the polarization decay time $T_{s 1}$ is inversely proportional to the exciton momentum scattering time, according to

$$
\frac{1}{T_{s 1}}=\left\langle\Omega(K)^{2}\right\rangle \tau^{*}
$$

leading to a motional narrowing effect as in the spin relaxation conduction electron mechanism described formerly by D'yakonov and Perel [5] (here, $\tau^{*}$ represents the exciton momentum elastic scattering time, a good estimation of which is given by the optical dephasing time $T_{2}$ [4], and the symbol \langle\rangle means the averaging on the created exciton states). For instance, in the QW presented in Fig. 1, we have measured (see below in Sec. 3) $T_{2} \approx 6$ ps at $10 \mathrm{~K}$. Under resonant excitation, we create an exciton wave packet whose typical extension in $K$-space is given by $K_{r}=\hbar^{-1} \sqrt{2 M_{X} \Gamma_{\mathrm{h}}}$, where $\Gamma_{\mathrm{h}}=2 \hbar / T_{2}$ is the exciton homogeneous spectral broadening, and $M_{X}$ - the exciton mass. The average in Eq. (5) is approximated by $\langle\Omega(K)\rangle \approx \Omega\left(K_{\Gamma}\right)$, which corresponds typically to $K_{\Gamma} \approx 3 \times 10^{5} \mathrm{~cm}^{-1}$ and $\Omega\left(K_{\Gamma}\right)$ - a few tens of $\mu \mathrm{eV}$, leading to good estimations of $T_{s 1}$ [4].

Figure $1 \mathrm{~b}$ displays the $P_{\operatorname{lin}}(t)$ dynamics after the resonant excitation of $X H$ by a linear pulse $\sigma^{x}$. We see that the characteristic decay time $T_{s 2}$, the so-called
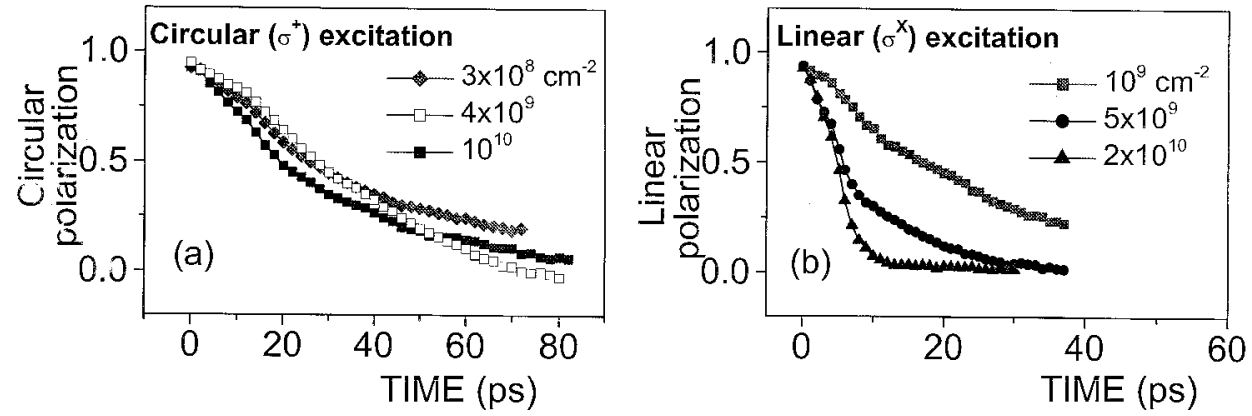

Fig. 1. GaAs $/ \mathrm{Al}_{x} \mathrm{Ga}_{1-x} \mathrm{As}(x=0.6)$ quantum well structure (well width $L_{\mathrm{w}}=10 \mathrm{~nm}$ ); (a) Circular polarization decay of an exciton gas resonantly excited by a short (1.4 ps) $\sigma^{+}$excitation pulse; (b) linear polarization dynamics for linear excitons excited by a $\sigma^{x}$ optical pulse, for different excitation powers. 
transverse spin relaxation time, depends now drastically on the exciton density, it increases while the exciton density decreases, reaching values of the same order as $T_{s 1}$, but remains below the theoretical limit predicted in [4]: $T_{s 2}=2 T_{s 1}$. As we developed previously [8], this is due to inter-exciton exchange which destroys the coherent superposition of the $|+1\rangle$ and $|-1\rangle$ components in linear excitons $|X\rangle=(|+1\rangle+|-1\rangle) / \sqrt{2}$ (or $|Y\rangle=(|+1\rangle+|-1\rangle) / \mathrm{i} \sqrt{2})$ generated by the $\sigma^{x}\left(\sigma^{y}\right)$ excitation pulse. We shall see later in Sec. 5 how the three-dimentional confinement achieved in quantum dots modifies completely this picture.

\section{Spin manipulations by temporal coherent control experiments in semiconductor nanostructures}

\subsection{Coherent control of exciton spin and alignment in quantum wells}

We show now how the control of exciton alignment and orientation can be achieved, i.e. how to fully control $S_{x}^{X}$ and $S_{z}^{X}$ by using polarized optical pulses. We use here the temporal coherent control approach, in which the structure is excited by a sequence of phase controlled polarized pulses. The behavior of the excitation populations or spin coherences can be monitored through reflectivity [9], transmission [10], or secondary emission (SE) [11, 12]. The interest of the coherent control approach lies in the fact that, choosing properly the intensities and polarizations of each pulse of the sequence, populations as well as spin coherences of the system can be manipulated within the photogenerated excitations dephasing time $T_{2}$. Using a cross linearly polarized sequence, we demonstrated that a quantum superposition of the states excited by each pulse of the sequence is really achieved in the low excitation field limit [13].

We show first temporal coherent control of the optical alignment of excitons in GaAs/AlGaAs quantum wells ( $L_{\mathrm{W}}=10 \mathrm{~nm}$ ) at low temperature $(T<10 \mathrm{~K})$, and low exciton densities (typically about $10^{9} \mathrm{~cm}^{-2}$ ). A sequence of two phase-locked 1.4 ps optical pulses, from a mode-locked Ti : sapphire laser, resonantly excites the heavy-hole excitons at the energy $E_{X H}$. The two pulses of equal intensities are circularly polarized, $\sigma^{+}$and $\sigma^{-}$, respectively, and are separated by a time delay $\tau=t_{1}+t_{2}\left(t_{1}=m h / E_{X H}-\right.$ coarse delay, equal to an integer multiple $m$ of the laser central component period $h / E_{X H} ; t_{2}$ - fine delay, allowing an accurate phase tuning between the two pulses). The time-resolved kinetics are recorded by up-converting the $\mathrm{SE}$ signal in a $\mathrm{LiIO}_{3}$ non-linear crystal with the output from an optical parametric oscillator synchronously pumped by the same Ti:Sa laser [11]. The time resolution (1.4 ps), is limited by the laser pulses duration.

Figure 2a shows the time dependence of the total SE and the linear polarization $P^{\mathrm{l}}(t)$ for $t_{2}=0$. The delay between the two excitation pulses is set to $t_{1}=6.6 \mathrm{ps}$, so that there is no temporal overlap between the two pulses. The excitation with the second laser pulse results in a sharp rise of the linear polarization of the excitonic luminescence which then decays with the characteristic time $T_{s 2}$. 
Obviously, this linear polarization originates from the interaction of the second pulse with the coherent excitonic polarization created in the sample by the first pulse. The linear polarization (measured 4 ps after the second pulse) is displayed as a function of $t_{2}$ in Fig. 2b. The observed oscillations are interpreted as due to the coherent superposition of the $|+1\rangle$ and $|-1\rangle$ heavy-hole exciton states generated respectively by the $\sigma^{+}$and $\sigma^{-}$pulses of the sequence, the phase factor between the two components corresponding to the one between the two optical pulses. The oscillation period corresponds to $h / E_{X H} \approx 2.6 \mathrm{fs}$. Figure $2 \mathrm{c}$ displays the minima and maxima of the linear polarization oscillations as a function of $t_{1}$. They decay with a characteristic time $T_{\mathrm{d}}=6 \pm 1 \mathrm{ps}$ at the temperature of $T=10 \mathrm{~K}$ and low exciton density $\left(\sim 10^{9} \mathrm{~cm}^{-2}\right)$. For a homogeneous $\mathrm{QW}$, it is clear that $T_{\mathrm{d}}$ can be identified to the heavy-hole exciton dephasing time $T_{2}$, as can be deduced from the optical Bloch equations of the system [13].
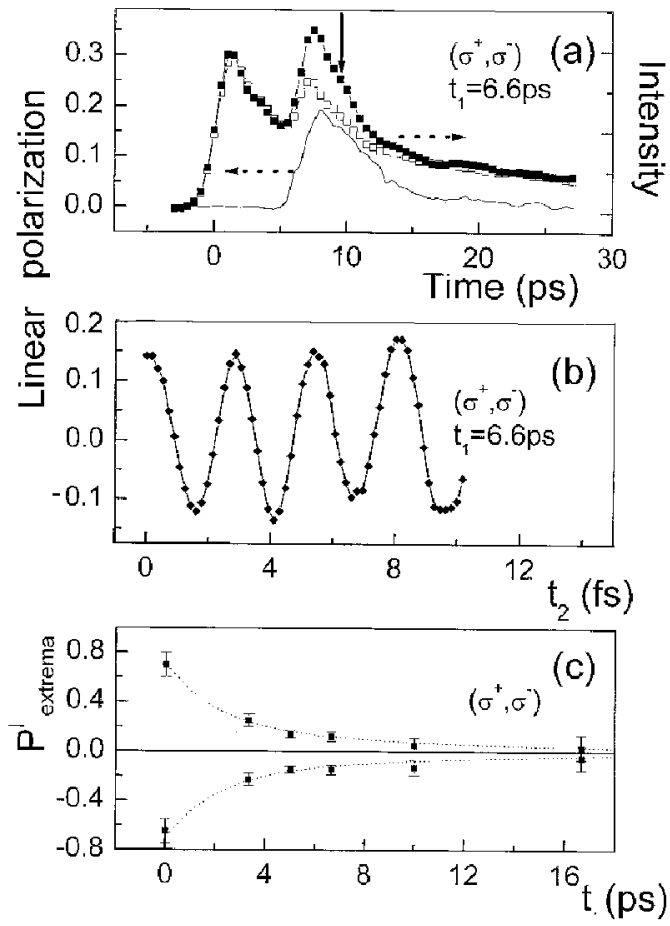

Fig. 2. The sequence configuration is $\left(\sigma^{+}, \sigma^{-}\right)$and $t_{1}=6.6 \mathrm{ps}$. (a) The time evolution of $I^{X}(\mathbf{a}), I^{Y}(\square)$ and the linear polarization $P_{\text {lin }}$ (full line) for $t_{2}=m h / E_{X H}$ (the back-scattered laser light from the sample surface is negligible). (b) The linear polarization $P^{\mathrm{l}}$ measured $4 \mathrm{ps}$ after the second excitation pulse [arrow in (a)] as a function of the fine temporal separation $t_{2}$ between the two excitation pulses. (c) The maxima and minima of the linear polarization oscillations as a function of $t_{1}$ (the dotted line is a guide for the eyes). 

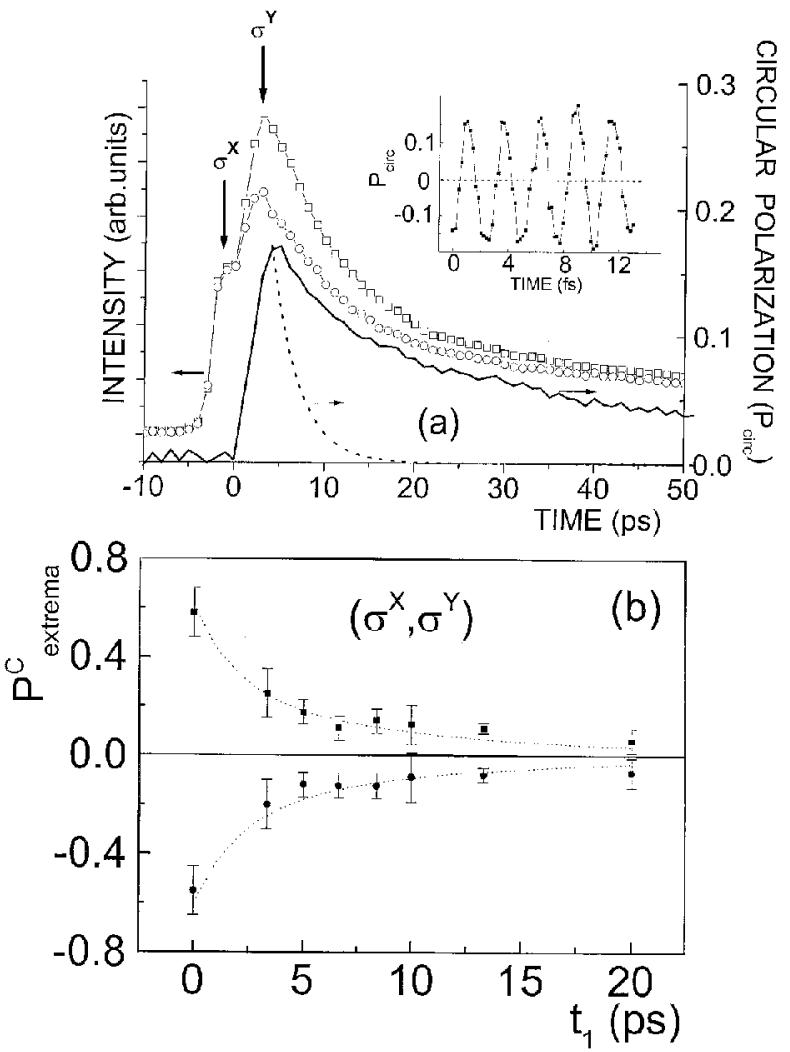

Fig. 3. The sequence configuration is $\left(\sigma^{x} \sigma^{y}\right)$ and $t_{1}=4 \mathrm{ps}$. (a) The time evolution of $I^{+}(\square), I^{-}(0)$ and the circular polarization $P_{\text {circ }}$ (full line) for $t_{2}=m h / E_{X H}$. Dotted line - expected $P_{\text {circ }}$ decay in the case of secondary emission dominated by optical interferences (see the text). Inset - the circular polarization $P_{\text {circ }}$ measured 4 ps after the second excitation pulse as a function of the fine temporal separation $t_{2}$ between the two excitation pulses. (b) The maxima and minima of the linear polarization oscillations as a function of $t_{1}$ (the dotted line is a guide for the eyes).

In order to achieve the coherent control of the exciton spin orientation, we use now a sequence of linearly polarized pulses $\left(\sigma^{x}, \sigma^{y}\right)$. Figure 3a shows the secondary emission intensity and circular polarization dynamics for pulses with the same phase $\left(t_{2}=0\right)$. The chosen coarse time delay $t_{1} \approx 4$ ps insures that there is no temporal overlap between the two pulses. Again we observe a fast increase in the emission circular polarization during the second pulse. The subsequent decay occurs with a characteristic time corresponding to the longitudinal spin relaxation time $T_{s 1}$. Clearly, this circular polarization is the consequence of the interaction of the second pulse with the coherent excitonic population created by the first one. The time dependence of $P_{\text {circ }}$ on $t_{2}$ measured 4 ps after the second pulse is displayed in the inset of Fig. 3a. The observed oscillations have again the period 
$h / E_{X H}$, and are interpreted as the various achieved coherent superpositions of the linear heavy exciton states $|X\rangle$ and $|Y\rangle$, with a relative phase factor identical to the one between the two excitation pulses. Figure $3 \mathrm{~b}$ shows that the oscillations amplitude decay is also $T_{\mathrm{d}}=6 \pm 1 \mathrm{ps}$ in the same conditions as previously.

The time range in which these experiments can be performed is indeed limited by the exciton optical dephasing time $T_{2}$. The observed decay time $T_{\mathrm{d}}$ can be identified with $T_{2}$ when the observation is performed in a non-specular direction [14]. In these conditions, $T_{2}$ decreases when the temperature is increased. The linear behavior of the associated $X H$ homogeneous broadening $\Gamma_{\mathrm{h}}=2 \hbar / T_{2}$ is the signature of the dephasing process due to acoustical phonon scattering, as seen in Fig. 4, and formerly observed in four wave mixing (FWM) experiments [15].

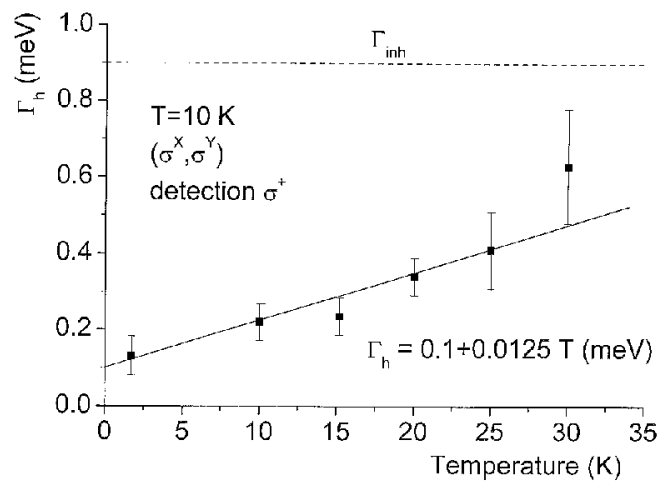

Fig. 4. Temperature dependence of $\Gamma \equiv 2 \hbar / T_{\mathrm{d}}$, and linear fit (solid line) to the experimental data. The dashed line represents the inhomogeneous broadening $\Gamma_{\text {inh }}$.

Note that the measured $\Gamma_{\mathrm{h}}$ value lies below the inhomogeneous broadening one $\Gamma_{\text {inh }}$ when the lattice temperature is below $30 \mathrm{~K}$. These results can be explained on the ground of recent experimental and theoretical works on secondary emission $[16,17]$. The latter is dominated, in our experimental conditions, by resonant Rayleigh scattering (RRS) induced by the interface structural disorder due to the molecular beam epitaxy growth process. The potential fluctuations experienced by the exciton center of mass imply their localization. In the case of strong localization, the individual excitonic dipoles emit coherent radiation in the observation direction with a phase factor which strongly fluctuates from site to site, determined by the local topology of the potential. The resulting interferences thus decay with the time $T_{2}$.

Figure 3 indicates that the excitonic emission remains circularly polarized after the second pulse, during a time which is much larger than the optical dephasing time $T_{2}$, i.e. when the emission is dominated by incoherent luminescence. This demonstrates that a real coherent superposition of $|X\rangle$ and $|Y\rangle$ states has been achieved for each individual dipole, and that the emission does not come from optical interferences of coherent emission radiated by localized dipoles either 
$|X\rangle$ or $|Y\rangle$ according to the site; in the latter case, the circular polarization decay time should coincides with $T_{2}$, and not $T_{s 1}$ (see the dotted line in Fig. 3a).

All these predictions can easily be deduced from the evolution equation of the exciton density operator

$$
\frac{\mathrm{d} \hat{\rho}}{\mathrm{d} t}=-\frac{\mathrm{i}}{\hbar}[H(t), \hat{\rho}]+\left.\frac{\mathrm{d} \hat{\rho}}{\mathrm{d} t}\right|_{\mathrm{relax}} .
$$

Here the system Hamiltonian is $H=H_{0}+H_{\text {int }}(t)$, where $H_{0}$ is the unperturbed Hamiltonian and $H_{\text {int }}(t)$ - the interaction Hamiltonian with light. Both Hamiltonians are restricted to the heavy-hole exciton subspace. In the dipolar approximation, $H_{\text {int }}(t)=-\widehat{\boldsymbol{\mu}} \cdot \boldsymbol{E}(t)$, where $\boldsymbol{\mu}$ is the dipolar operator for the heavy-hole exciton, and $\boldsymbol{E}(t)=\boldsymbol{E}_{1}(t)+\boldsymbol{E}_{2}(t-\tau)$ is the electric field amplitude $\left(\tau=t_{1}+t_{2}\right)$.

The phenomenological terms $\left.\frac{d \widehat{p}}{d t}\right|_{\text {relax }}$ describe the relaxation of the system towards the thermodynamical equilibrium. Within the relaxation time approximation, and for a homogeneous system, the relaxation of population terms writes in the basis $\{|\varnothing\rangle,|+1\rangle,|-1\rangle\}$

$$
\begin{aligned}
& \left.\frac{\mathrm{d} \rho_{ \pm 1, \pm 1}}{\mathrm{~d} t}\right|_{\text {relax }}=-\frac{1}{T_{1}} \rho_{ \pm 1, \pm 1}-\frac{1}{2 T_{s 1}}\left(\rho_{ \pm 1, \pm 1}-\rho_{\mp 1, \mp 1}\right), \\
& \left.\frac{\mathrm{d} \rho_{\oslash, \oslash}}{\mathrm{d} t}\right|_{\text {relax }}=\frac{1}{T_{1}}\left(\rho_{1,1}+\rho_{-1,-1}\right),
\end{aligned}
$$

where $|\oslash\rangle$ represents the QW fundamental state (no excitons), and $T_{1}$ is the intrinsic radiative exciton lifetime. The coherences decay is given by the non-diagonal relaxation terms

$$
\begin{aligned}
& \left.\frac{\mathrm{d} \rho_{-1,1}}{\mathrm{~d} t}\right|_{\text {relax }}=-\left(\frac{1}{T_{1}}+\frac{1}{T_{s 2}}\right) \rho_{-1,1}, \\
& \left.\frac{\mathrm{d} \rho_{\oslash, \pm 1}}{\mathrm{~d} t}\right|_{\text {relax }}=-\frac{1}{T_{2}} \rho_{\oslash, \pm 1} .
\end{aligned}
$$

The optical dephasing time of optically active excitons is related to the lifetime by $1 / T_{2}=1 /\left(2 T_{1}\right)+1 / T_{2}^{\prime}$, where $T_{2}^{\prime}$ represents the characteristic time of pure (elastic) dephasing processes. Corresponding expressions can be obtained in the $\{|\oslash\rangle,|X\rangle,|Y\rangle\}$ basis. The equation of the density matrix can be solved analytically in the case where $\tau, T_{2} \ll T_{1}, T_{s 2}$. The quantum well interband polarization is given by $\boldsymbol{P}=\operatorname{Tr}(\widehat{\rho \mu})$. For a $\left(\sigma^{X}, \sigma^{Y}\right)$ excitation sequence applied to a homogeneous system, we obtain, to the first order approximation with respect to the electric field amplitudes and with equal intensities short pulses [13]

$$
P_{\text {circ }}(t)=\frac{2 \exp \left(-\tau / T_{2}\right) \sin \left(\omega_{H} \tau\right)}{\exp \left(-\tau / T_{1}\right)+1} \exp \left[-(t-\tau) / T_{s 1}\right], \quad(t>\tau)
$$

More details are given in Ref. [14] in the case of inhomogeneous systems.

Although the feasibility of coherent control experiments is well established, it is highly desirable, with respect to potential applications, to investigate systems where the exciton spin relaxation and optical dephasing times are much longer. 


\subsection{Coherent control of exciton-polaritons spin and alignment in microcavities}

Semiconductor microcavities in a strong coupling regime offer a unique possibility to control both spin relaxation and dephasing time. The presented structures, elaborated by molecular beam epitaxy, consist in a high finesse planar optical cavities delimited by two Bragg mirrors made of alternating $\mathrm{Al}_{y} \mathrm{Ga}_{1-y} \mathrm{As} / \mathrm{AlAs}$ quarter wave plates; one or several $\operatorname{In}_{x} \mathrm{Ga}_{1-x}$ As quantum wells are inserted during the growth at the antinodes of the electromagnetic field of the cavity. As demonstrated by Weisbuch et al. [18], when the cavity finesse is high enough, exciton and cavity modes with the same wave vector $k$ and angular momentum $J_{z}$ [19] couple together, thus leading to a mixed quasi-particle, the so-called cavity-polaritons. The new eigenmodes consist in two branches, which dispersion is determined by

$$
\begin{aligned}
& E_{L B(U B)}(K)=\frac{1}{2}\left[E_{C}(K)+E_{X H}(K)\right] \\
& \mp \frac{1}{2} \sqrt{\left[E_{C}(K)-E_{X H}(K)\right]^{2}+\left(\hbar \Omega_{\mathrm{R}}\right)^{2}},
\end{aligned}
$$

where $E_{C}(K)$ is the photon cavity mode and $\Omega_{\mathrm{R}}$ is the vacuum Rabi splitting, characterizing the exciton-photon coupling strength. The index $U B$ and $L B$ denote the upper and lower polariton branch, respectively). The eigenstates are given by

$$
\begin{aligned}
\left|J_{z}, \boldsymbol{K}\right\rangle^{L B} & =+X(K)\left|J_{z}, \boldsymbol{K}\right\rangle^{X}+C(K)\left|J_{z}, \boldsymbol{K}\right\rangle^{C} \\
\left|J_{z}, \boldsymbol{K}\right\rangle^{U B} & =-C(K)\left|J_{z}, \boldsymbol{K}\right\rangle^{X}+X(K)\left|J_{z}, \boldsymbol{K}\right\rangle^{C}
\end{aligned}
$$

Here, $X(K)$ and $C(K)$ are the Hopfield coefficients [20]:

$$
\begin{aligned}
& X(K)=\left[1+\left(\frac{\hbar \Omega_{\mathrm{R}}}{E_{L B}(K)-E_{C}(K)}\right)^{2}\right]^{-\frac{1}{2}}, \\
& C(K)=-\left[1+\left(\frac{E_{L B}(K)-E_{C}(K)}{\hbar \Omega_{\mathrm{R}}}\right)^{2}\right]^{-\frac{1}{2}}
\end{aligned}
$$

and $\left|J_{z}, \boldsymbol{K}\right\rangle^{A}$ represent one-particle states of angular momentum $J_{z}= \pm 1$ and wave vector $\boldsymbol{K}$ for excitons, cavity-photons, polaritons $[A=X, C, L B(U B)$, respectively]. Figure 5a,b shows the polariton energy position, exciton and polariton weights as a function of the cavity detuning $\delta \equiv\left[E_{C}(0)-E_{X H}(0)\right]$ with respect to the exciton mode in $K=0$. Figure $5 \mathrm{c}$ shows the dispersion curves for $\delta=0^{*}$.

\subsection{Polariton spin relaxation in microcavities}

The polariton angular momentum corresponds to the eigenstates of the operator $\hat{J}_{Z}=\hat{J}_{z}^{X} \oplus \hat{J}_{z}^{C}$, where $\hat{J}_{z}^{X}$ and $\hat{J}_{z}^{C}$ represent the projection on the growth

*In the GaInAs/GaAs QW microcavities presented here, the biaxial strain due to lattice mismatch in the QW repels the light-hole excitons by a few tens of meV to higher energies, so that they can be ignored in the spectral domain corresponding to heavy-hole excitons-polaritons. 

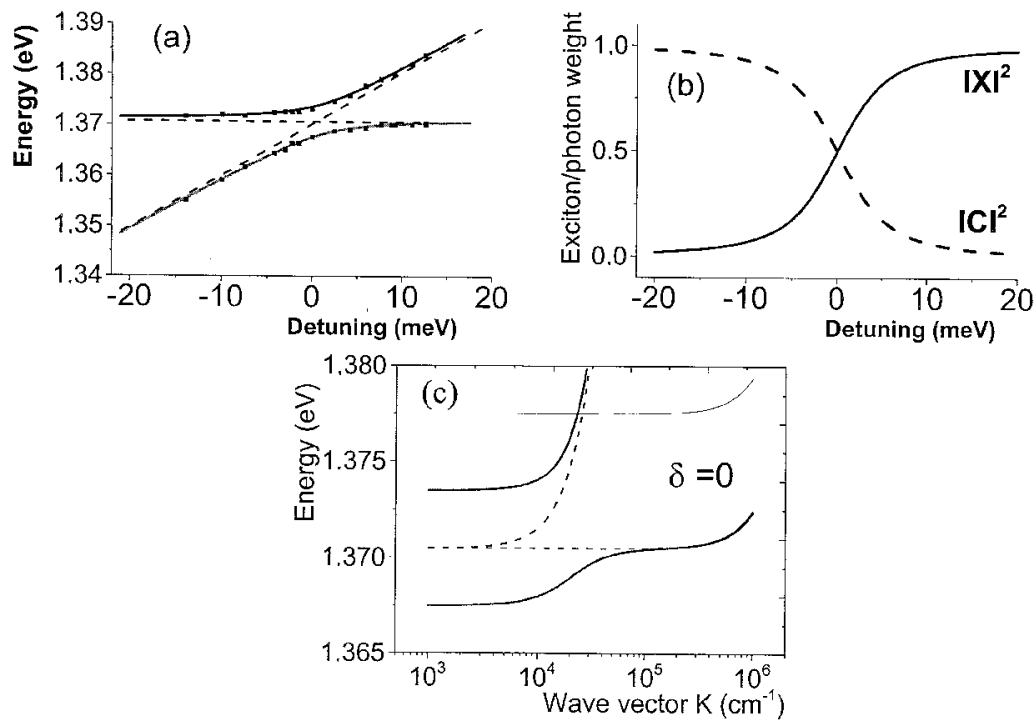

Fig. 5. (a) Energies of the polariton modes, measured in a $\mathrm{cw}$ photoluminescence experiment (PLE) as a function of the cavity detuning $\delta$ (the same excitation geometry as the time-resolved experiments is used). The full lines correspond to the calculated energies of the exciton-photon mixed states (see the text). The dashed lines are the uncoupled cavity mode and exciton energies. (b) Calculated exciton $\left(|X|^{2}\right.$, solid line) and cavity photon mode $\left(|C|^{2}\right.$, dashed line) weight of the lower branch polariton state as a function of $\delta$. (c) Calculated polariton dispersion curves at $\delta=0$ (bold line - polariton branches, dashed lines — uncoupled modes, solid line - QW gap).

axis of the exciton and photon angular momentum (only optically active $J^{X}=1$ are indeed coupled to light). The polariton states in each branch are twice degenerated, the corresponding two states having a \pm 1 angular momentum (in units of $\hbar$ ). We can thus define a polariton effective spin, and, in analogy with the definitions adopted in bare quantum wells, it is possible to define polariton longitudinal (transverse) spin relaxation times as the decay of the secondary emission circular (linear) polarization degree.

In the following, the polariton lower branch is excited resonantly at a small incidence angle $\left(\sim 8^{\circ}\right.$, which corresponds to an initial in plane wave vector of $K_{\mathrm{p}} \approx 10^{4} \mathrm{~cm}^{-1}$ ), and the detection is performed normal to the microcavity surface. The cavities are grown with a wedge, so that it is possible to adjust the detuning by moving the excitation spot on the sample surface. The excitation power is weak, in order to avoid stimulated parametric processes [21, 22]. Figure 6 displays the circular polarization decay of the microcavity SE under a single $\sigma^{+}$excitation for two cavity detunings $\left(\delta=0\right.$ and $\delta=-3 \mathrm{meV}$ ). We find $T_{s 1} \approx 50 \mathrm{ps}$ for $\delta=0$ and $T_{s 1} \approx 1 \mathrm{~ns}$ for $\delta=-3 \mathrm{meV}$ (in the latter case, the dynamics cannot be measured beyond $50 \mathrm{ps}$ because of the short polariton lifetime). It is clear 

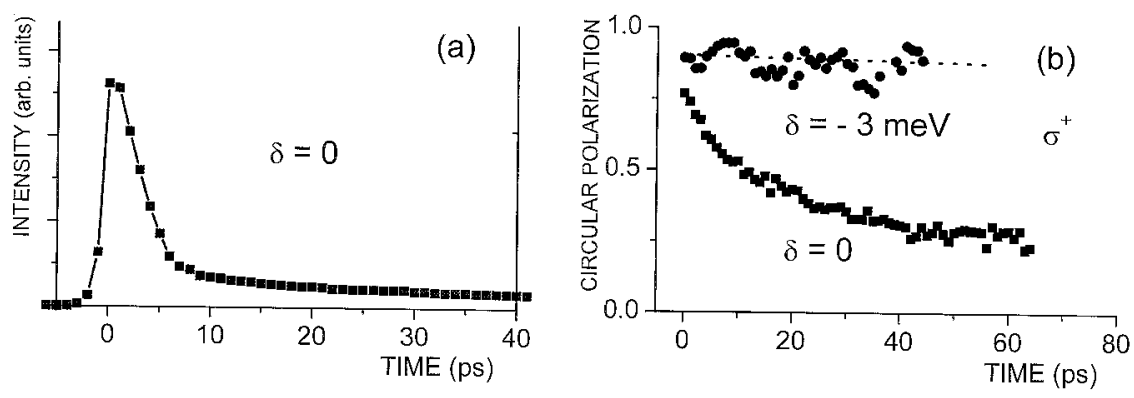

Fig. 6. (a) Total intensity decay at $\delta=0$ and (b) circular polarization decays of the microcavity $\mathrm{MC1}$ for $\delta=-3 \mathrm{meV}(\bullet)$ and $\delta=0(\mathbf{a})$ following a $\sigma^{+}$excitation pulse.

here that the efficiency of the spin relaxation process, which is governed by the long range electron-hole exchange interaction within the exciton component of the polariton, decreases when the exciton character of the polariton decreases. Taking into account that $\Omega \ll \Omega_{\mathrm{R}}$, we have $\Omega^{L B}(K)=|X(K)|^{2} \Omega(K)$. Finally, in analogy with (5), we obtain for the polariton spin relaxation time

$$
\frac{1}{T_{s 1}^{L B}}=\left\langle\left(\Omega^{L B}(K)\right)^{2}\right\rangle \tau_{L B}^{*}
$$

Here, $\tau_{L B}^{*}$ is the polariton momentum relaxation time due to elastic scattering processes. In our experiments, the initial polariton wave vector is about $K_{\mathrm{p}} \approx$ $10^{4} \mathrm{~cm}^{-1}$, corresponding to a squared long range exchange interaction $\left[\Omega^{L B}\left(K_{\mathrm{p}}\right)\right]^{2}$ about three orders of magnitude lower than in the previous case of bare excitons. On the other hand, due to the strong energy dispersion in the excited region at negative detuning, the elastic scattering time becomes longer by an order of magnitude with respect to the bare exciton one, as seen below ( $T_{2}^{\prime} \approx 20 \mathrm{ps}$ for $\delta=-3 \mathrm{meV}$ ), so that the dephasing time is mainly determined by the polariton escape time from the cavity. We have measured $T_{1} \approx 2.5 \mathrm{ps}$, so that $1 / T_{2}=$ $1 /\left(2 T_{1}\right)+1 / T_{2}^{\prime} \approx 1 /\left(2 T_{1}\right)$. Taking $T_{2}^{\prime}$ as an estimate of $\tau^{*}$ as is usually done, we obtain thus for $\delta=-3 \mathrm{meV} \tau^{*} \approx 20 \mathrm{ps}$ and $\Gamma_{\mathrm{h}} \approx \hbar / T_{1} \approx 0.3 \mathrm{meV}$. However, due to the small polariton mass (about $10^{-4}$ times the exciton mass) the extension in $\boldsymbol{K}$-space of the created polariton wave packet is small with respect to $K_{\mathrm{p}}\left(K_{\Gamma} \ll\right.$ $\left.K_{\mathrm{p}}\right)$, so we can use the following approximation: $\left\langle\left(\Omega^{L B}(K)\right)^{2}\right\rangle \approx\left(\Omega^{L B}\left(K_{\mathrm{p}}\right)\right)^{2}$. The polariton spin relaxation time $T_{s 1}^{L B}$ is thus more than two orders of magnitude larger than the bare exciton one $T_{s 1}$. This results in the blocking of the longitudinal polariton spin relaxation at negative detuning as it is observed in Fig. 6. Finally, the same blocking effect can be obtained at negative detuning for linearly polarized polaritons, when polariton mutual exchange interaction is quenched [23].

\subsection{Coherent control of polariton alignment and angular momentum}

We extend now the method applied in Sec. 3.1 to quantum well microcavities, and show how the spin orientation, alignment (and density) of polaritons 
can be coherently manipulated. In addition, polariton dephasing times can be estimated [24, 25]. Let us consider for instance a sequence of two optical pulses of opposite helicity $\left(\sigma^{+}, \sigma^{-}\right)$and excite resonantly the $L B$ polariton for a cavity detuning $\delta=0$. Figure 7 a displays the time dependence of the total SE when the coarse time delay between the two excitation pulses $\left(\sigma^{+}, \sigma^{-}\right)$is $t_{1}=6 \mathrm{ps}$, so that there is no temporal overlap between the two pulses. Nevertheless, the excitation with the second pulse results in a linearly polarized SE. As shown in the inset of Fig. 7a, this linear polarization oscillates at the pulsation $\omega=E_{L B} / \hbar$ as a function of the fine temporal delay $t_{2}$ (this recording, as all the similar data in this section, is systematically taken just after $(\sim 1 \mathrm{ps})$ the second pulse). These oscillations reflect the rotation of the orientation of the linear exciton-polaritons in the cavity plane, when $t_{2}$ is varied. The oscillations amplitude decay time $T_{\mathrm{d}}$ is measured in Fig. $7 \mathrm{~b}$, where we find $T_{\mathrm{d}}=3.2 \pm 1 \mathrm{ps}$.
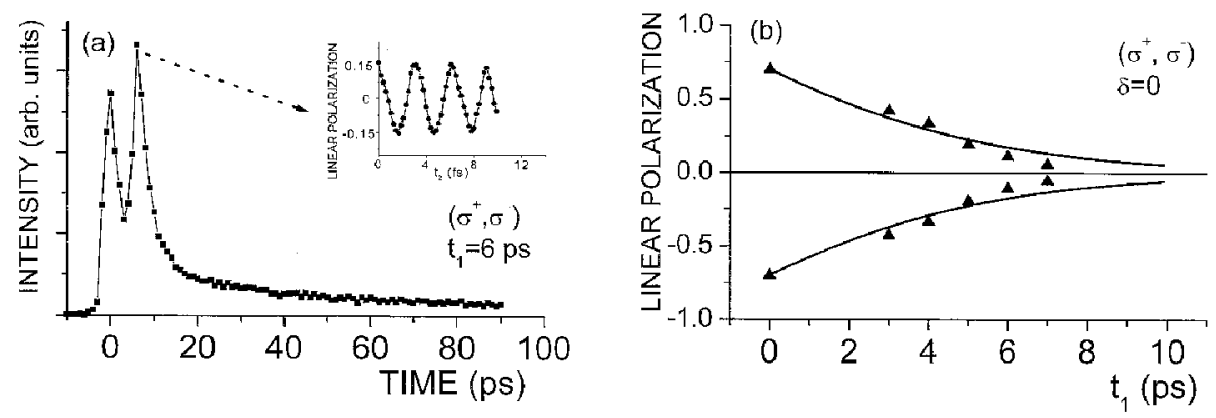

Fig. 7. The cavity detuning is $\delta=0$. (a) Time evolution of the total SE ( $)$ after a $\left(\sigma^{+}, \sigma^{-}\right)$excitation sequence: $t_{1}=6 \mathrm{ps}$ and $t_{2}=0$. Inset - linear polarization as a function of the fine temporal separation $t_{2}$ between the two excitation pulses. (b) The maxima and minima of the linear polarization oscillations as a function of $t_{1}$. The solid line is the fit using Eq. (14) (see the text).

Coherent control of microcavity polariton spin can be achieved with a sequence of two linearly cross-polarized laser pulses $\left(\sigma^{x}, \sigma^{y}\right)$. The excitation with the second pulse results in a circularly polarized SE, as illustrated in Fig. 8 for $t_{1}=4$ ps. The amplitude decay of this circular polarization oscillations as a function of $t_{1}$ yields also the measurement of $T_{2}$.

In microcavities, the polarization oscillations observed in Figs. 7a and 8 demonstrate that the coherent emission from the $K \cong 0$ exciton-polariton states can be obtained from an off-normal excitation direction. This fact was previously observed by Norris et al. [26] in interferometric pump-probe experiments performed in non specular directions. We interpret this as the result of the coupling by the disorder induced potential of $K_{\mathrm{p}}$ and $K \cong 0$ polariton states through their excitonic component [27], which leads to inhomogeneous broadening of polariton 


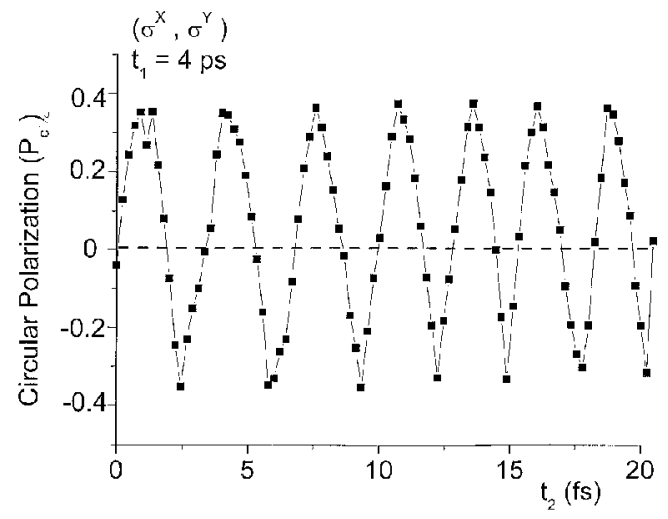

Fig. 8. The cavity detuning is $\delta=0$ and the configuration is $\left(\sigma^{x}, \sigma^{y}\right)$. Circular polarization as a function of the fine temporal separation $t_{2}$ between the two excitation pulses $\left(t_{1}=4 \mathrm{ps}\right)$.

states and the possibility of resonant Rayleigh scattering. We can thus conclude that, due to the RRS nature of the detected signal, the polarization oscillations decay time $T_{\mathrm{d}}$ observed in our experiments is the polariton dephasing time $T_{2}$. For microcavities with a very low disorder, the energy conservation and dispersion effects should in principle lead to RRS emission directions located on the surface of a cone centered around the normal direction to the microcavity, the top angle of which is determined by the excitation light angle with respect to that normal [28]. However, the energy dispersion between $K_{\mathrm{p}} \cong 10^{4} \mathrm{~cm}^{-1}$ and $K \cong 0$ is, in all cases investigated here, smaller than the polariton states broadening ${ }^{\dagger}$, so that elastic light scattering becomes possible out of the above defined conical surface.

We study now the dependence of the optical dephasing time on the cavity detuning. Using e.g. a $\left(\sigma^{+}, \sigma^{-}\right)$pulse sequence, it can be shown that just after the second pulse $(t=\tau+0)$, the SE linear polarization writes (see Annex 1)

$$
P_{\text {lin }}(\tau)=P_{\text {lin }}(\tau=0) \frac{2 \exp \left(-\tau / T_{2}\right)}{1+\exp \left(\tau / T_{1}\right)} \cos \left(\frac{E_{L B} \tau}{\hbar}\right) .
$$

This expression is valid when the polariton states can be considered as homogeneous. However its validity can be extended here in the case of inhomogeneously broadened polariton states [5]. The decay of the oscillations amplitude, which yields the measurement of $T_{2}$, is displayed in Figs. $7 \mathrm{~b}$ and 9 for three cavity detunings. Using the $T_{1}$ values measured from the intensity decay in single pulse experiments $T_{1}=2.5 \pm 1 \mathrm{ps}, 3.5 \pm 1 \mathrm{ps}$, and $6 \pm 1 \mathrm{ps}$, we find, using (14), $T_{2}=4 \pm 1 \mathrm{ps}$, $3.2 \pm 1 \mathrm{ps}$, and $2.5 \pm 1 \mathrm{ps}$, for $\delta=-3,0$ and $3 \mathrm{meV}$, respectively. The trend is in agreement with the result of the FWM experiment performed by Wang et al. [29], who observed that the FWM signal decay was faster at the resonance $(\delta=0)$ than for negative detuning. This trend can be interpreted from the simple expression

\footnotetext{
${ }^{\dagger}$ When $-3 \leq \delta \leq+3 \mathrm{meV}$, we calculate that $E\left(K_{\mathrm{p}}\right)-E(0)<0.7 \mathrm{meV}$ while the polariton linewidth $\Gamma$ verifies $\Gamma>1.2 \mathrm{meV}$ for $0 \leq K, K_{\mathrm{p}} \leq 10^{4} \mathrm{~cm}^{-1}$.
} 


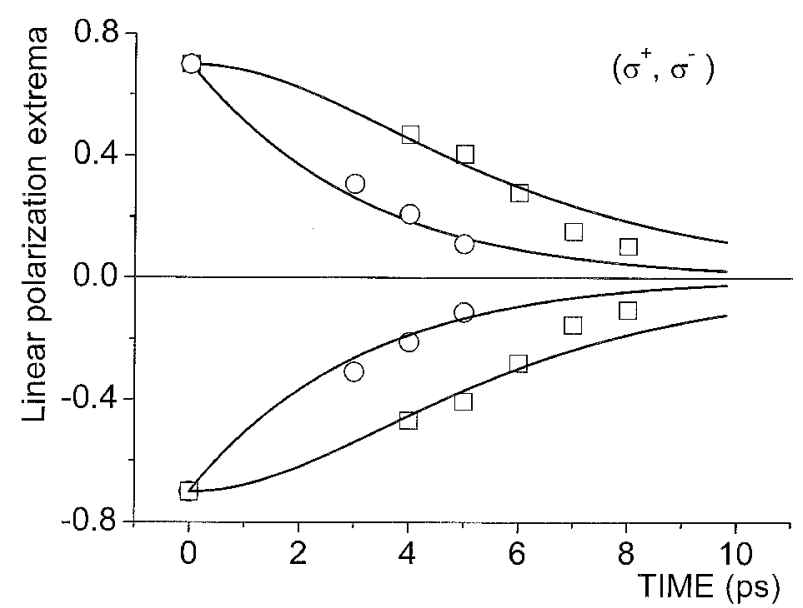

Fig. 9. The cavity detuning dependence of $T_{2}$. The minima and maxima of the linear polarization oscillations as a function of $t_{1}$ for $\delta=-3(\square)$ and $+3 \mathrm{meV}(0)$, respectively. The solid lines are the fit using the model (14) described in the text.

[15]: $1 / T_{2}=1 /\left(2 T_{1}\right)+1 / T_{2}^{\prime}$, where $T_{2}^{\prime}$ represents the characteristic time of pure dephasing processes. For the negative detuning $\delta=-3 \mathrm{meV}, T_{2}$ is very close to $2 T_{1}$ which indicates that, when the photon character of the polariton state dominates, the optical dephasing time is determined by the cavity photon lifetime. The contribution of pure dephasing processes is then small. In contrast, for the positive detuning $\delta=+3 \mathrm{meV}$, the lifetime is no longer responsible for the phase coherence loss since $T_{1} \sim 6$ ps and $T_{2} \sim 2.5 \mathrm{ps}$. Clearly, when the polariton has a dominant exciton character, the optical dephasing is due to the pure dephasing processes represented by $T_{2}^{\prime}$. In this case, the measured $T_{2}$ value is close to the values reported for heavy-hole excitons in bare QWs of equivalent quality [30]. Our measured dependence of $T_{2}$ on $\delta[24,25]$ is in qualitative agreement with the calculations of the homogeneous broadening $\Gamma_{L B(U B)}$ of the polariton modes by Savona et al. [31], which show that the contribution of acoustical phonons to $\Gamma_{L B}$ is one order of magnitude smaller (at $\delta=0$ ) than the value (of 2 to $12 \mu \mathrm{eV} / \mathrm{K}$ ) for bare QW excitons.

\section{Exciton spin quantum beats under magnetic field in semiconductor nanostructures}

Another possibility for manipulating exciton spin is naturally offered by the use of magnetic fields [32, 33]. In quantum wells, a transverse magnetic field for instance essentially couples the $|+1\rangle$ to the $|+2\rangle$ (and symmetrically the $|-1\rangle$ to the $|-2\rangle$ ) exciton states through its conduction electron component, while a longitudinal field provides the usual Zeeman splitting between $J_{z}= \pm 1$ (and $J_{z}= \pm 2$ ) states (the angular momenta unit is $\hbar$ ). Although it would be necessary to use transient pulses of magnetic field in order to achieve a given coherent 
superposition of quantum states, thus achieving some quantum function, we shall here only consider static fields, in order to investigate the potentialities of such an approach.

Under a longitudinal magnetic field $B_{z}$, and using a resonant $\sigma^{x}$ optical pulse at small incidence angle (Faraday configuration), the linearly polarized components are recorded. Figure 10 displays the linear polarization oscillations of the secondary

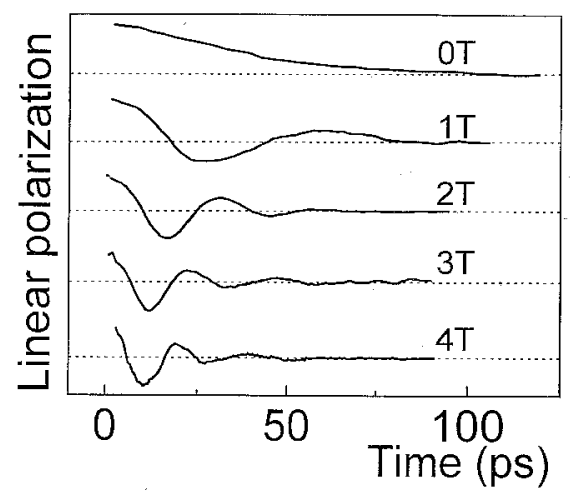

Fig. 10. GaAS $/ \mathrm{Al}_{0.3} \mathrm{Ga}_{0.7}$ As quantum well structure $\left(L_{\mathrm{w}}=4.8 \mathrm{~nm}\right)$ in a longitudinal magnetic field, linear polarization oscillations following a $\sigma^{x}$ excitation pulse revealing the exciton spin beats under a longitudinal magnetic field. The temperature is $1.7 \mathrm{~K}$.

emission, observed at low temperature, and low density $\left(\sim 10^{9} \mathrm{~cm}^{-2}\right)$, revealing the exciton spin beats $[34,35]$. The effective exciton Hamiltonian writes, in the $J=1$ subspace: $H_{B}=\left(g_{\mathrm{e}, \|}+g_{\mathrm{h}, \|}\right) \mu_{\mathrm{B}} B_{z} \hat{S}_{z}^{X} \quad\left(g_{\mathrm{e}, \|}\right.$ and $g_{\mathrm{h}, \|}$ are the longitudinal electron and hole Landé $g$-factors). The temporal evolution of the exciton spin $\boldsymbol{S}^{X}$ is simply given by the following expression:

$$
\frac{\mathrm{d} \boldsymbol{S}^{X}}{\mathrm{~d} t}=\Omega_{B} \boldsymbol{e}_{z} \times \boldsymbol{S}^{X}-\frac{\boldsymbol{S}^{X}}{T_{s 2}},
$$

where $\hbar \Omega_{B}=\left(g_{\mathrm{e}, \|}+g_{\mathrm{h}, \|}\right) \mu_{\mathrm{B}} B_{z}$ represents the Zeeman splitting of the $J=1$ exciton states. The initial condition is given by $\boldsymbol{S}^{X}(0)=(1 / 2) \boldsymbol{e}_{x}\left(P_{\operatorname{lin}}(0)=2 S_{x}=1\right)$. The exciton spin precesses around $\boldsymbol{e}_{z}$ and damps with $T_{s 2}[34,35]$. The linear polarization oscillations disappear in a nonresonant excitation, or when the exciton temperature or density is raised.

Under transverse magnetic field, on the other hand, the electron-hole exchange interaction $\delta_{X}$ deeply modifies the oscillatory behavior of the exciton luminescence under resonant excitation. We use now a circularly polarized $\sigma^{+}$excitation light pulse (Voigt configuration), and the emission components of opposite helicities are detected. Under resonant excitation (Fig. 11b), the emission copolarized with the laser $\left(I^{+}\right)$oscillates, while the counter polarized one $\left(I^{-}\right)$smoothly increases, practically without oscillations, due to exciton spin relaxation [35]. The 


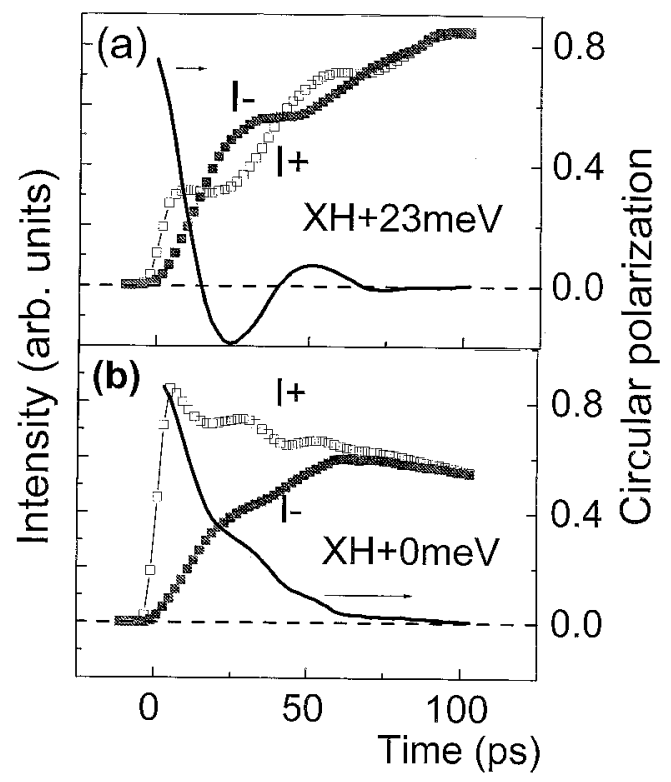

Fig. 11. $\mathrm{GaAs} / \mathrm{Al}_{0.3} \mathrm{Ga}_{0.7} \mathrm{As}$ quantum well structure $\left(L_{\mathrm{w}}=3 \mathrm{~nm}\right)$ in a transverse magnetic field $B_{x}=2.8 \mathrm{~T}$. Intensity and polarization dynamics after a $\sigma^{+}$excitation pulse: (a) the excitation energy is above the quantum well gap; (b) the excitation is resonant with $X H$. The temperature is $1.7 \mathrm{~K}$.

polarization decreases with the characteristic time $T_{s 1} \approx 30 \mathrm{ps}$. Assuming that the heavy-hole states which participate to the electron-hole pair manifold which constitute the $X H$ exciton are pure $| \pm 3 / 2\rangle$ angular momentum (which is achieved when the heavy-light exciton splitting is larger than the $X H$ binding energy), the dominant terms in the electron-hole effective spin Hamitonian are as follows [36]:

$$
H_{B}=\hbar \omega_{\mathrm{L}} \hat{s}_{x}-\frac{2}{3} \delta_{x} \hat{j}_{z} \hat{s}_{z},
$$

where $\hbar \omega_{\mathrm{L}}=g_{\mathrm{e}, \perp} \mu_{\mathrm{B}} B_{x}$ is the electron Larmor pulsation, $g_{\mathrm{e}, \perp}$, is the electron transverse $g$-factor (the hole transverse $g$-factor, generally one order of magnitude lower than $g_{e, \perp}$, is neglected here [37]). The magnetic field mixes $|+1\rangle$ and $|+2\rangle$ exciton states, the splitting between the new eigenstates $\left|\psi_{+}\right\rangle$and $\left|\psi_{-}\right\rangle$being now: $\hbar \Omega_{B}=\sqrt{\left(\delta_{X}\right)^{2}+\left(\hbar \omega_{\mathrm{L}}\right)^{2}}$ which under weak magnetic field approaches $\delta_{X}$. As the $\sigma^{+}$excitation pulse creates a coherent superposition of $\left|\psi_{+}\right\rangle$and $\left|\psi_{-}\right\rangle$states, the oscillation pulsation observed on $I^{+}$corresponds to $\Omega_{B}$. The modulation amplitude of the $I^{+}$oscillations is limited by the factor $\left(\omega_{\mathrm{L}} / \Omega\right)^{2}$ determined by the exciton state mixing, thus explaining why these oscillations can only be detected when $\hbar \omega_{\mathrm{L}}$ becomes comparable to $\delta_{X}$.

Under non-resonant excitation, the emission still displays an oscillatory behavior [35, 36, 38], which contrasts with similar experiments performed under longitudinal magnetic field, as reported above. The $I^{+}$and $I^{-}$oscillate now with 
opposite phases (see Fig. 11a), at the Larmor $\omega_{\mathrm{L}}$ pulsation of the electron. Although the detection energy corresponds to $X H$, at a moderate exciton density $\left(\sim 10^{9} \mathrm{~cm}^{-2}\right)$, the exchange energy $\delta_{X}$ does not manifest here any more. However, the circular polarization decays with a time which nearly coincides with the longitudinal exciton spin relaxation time $T_{s 1}$. The interpretation in terms of Larmor precession of the electron alone is not surprising at a high electron-hole pair density $\left(\sim 10^{11} \mathrm{~cm}^{-2}\right)$, or at high temperatures $(\sim 200 \mathrm{~K})$, when the excitons are not stable quasi-particles, and the recombination originates from unbound electron-hole pairs; it becomes puzzling when excitons are present, and the detected luminescence comes from the exciton luminescence [39].

We have shown that the hole spin stability completely determines these two extreme behaviors [36]. For the electron, the short range exchange interaction with the hole is equivalent to an effective magnetic field, denoted the "exchange field" orientated along $O z$. If the hole spin is stable, the electron spin Hamiltonian is equivalent to $H_{B}=\hbar \omega_{\mathrm{L}} \hat{s}_{x}+\Omega_{\mathrm{exc}} \hat{s}_{z}$, where $\Omega_{\mathrm{exc}}=-\frac{2}{3} \delta_{X}\left\langle j_{z}\right\rangle$, so that the electron spin $s=\langle\hat{s}\rangle$ precesses according to

$$
\frac{\mathrm{d} s}{\mathrm{~d} t}=\left(\omega_{\mathrm{L}} \boldsymbol{e}_{\boldsymbol{x}}+\Omega_{\mathrm{exc}} \boldsymbol{e}_{x}\right) \times \boldsymbol{s} .
$$

For $\sigma^{+}$excitation, $s(0)=(-1 / 2) \boldsymbol{e}_{\boldsymbol{z}}$, and $\Omega_{\mathrm{exc}}=-\delta_{X}$. If, on the contrary, the hole spin relaxes in a time $\tau_{\mathrm{h}}$ smaller than $\hbar / \Omega$, the electron does not "feel" any more the hole exchange field $\left(\left\langle\hat{j}_{z}\right\rangle=0\right)$. As a consequence, the polarization quantum beats occur in the excitonic luminescence at the Larmor pulsation $\omega_{\mathrm{L}}$, in a similar way to the case of free electrons in unbound electron-hole pairs observed previously $[38,39]$. In the general case, the electron spin is coupled to a variable characterizing the electron-hole spin correlation $\boldsymbol{Q}=2 / 3\left\langle\hat{j}_{z} \hat{\boldsymbol{s}}\right\rangle$. The electron spin evolution is then determined by the following equations [36]:

$$
\begin{aligned}
& \frac{\mathrm{d} s}{\mathrm{~d} t}=\omega_{\mathrm{L}} \boldsymbol{e}_{x} \times s-(\delta / \hbar) \boldsymbol{e}_{z} \times \boldsymbol{Q}, \\
& \frac{\mathrm{d} \boldsymbol{Q}}{\mathrm{d} t}=\omega_{\mathrm{L}} \boldsymbol{e}_{\boldsymbol{x}} \times \boldsymbol{Q}-(\delta / \hbar) \boldsymbol{e}_{z z} \times s-\frac{\boldsymbol{Q}}{\tau_{\mathrm{h}}}, \\
& \frac{\mathrm{d} j_{z}}{\mathrm{~d} t}=-\frac{j_{z}}{\tau_{\mathrm{h}}},
\end{aligned}
$$

where the damping terms due to hole spin relaxation have been only retained. These equations allow us to describe the intermediate cases. From direct measurements of $\tau_{\mathrm{h}}$ [40], we have learnt that the condition to observe electron spin beats $\left(\tau_{\mathrm{h}}<\hbar / \Omega\right)$ is currently satisfied when one excites a quantum well non-resonantly with $X H$, whatever is its width. The hole spin instability is here in relation with the fact that the exciton temperature is higher than the lattice temperature. The observation of exciton beats in narrow quantum wells under resonant excitation proves that the hole spin is stabilized in a cold two-dimensional exciton gas. Note 

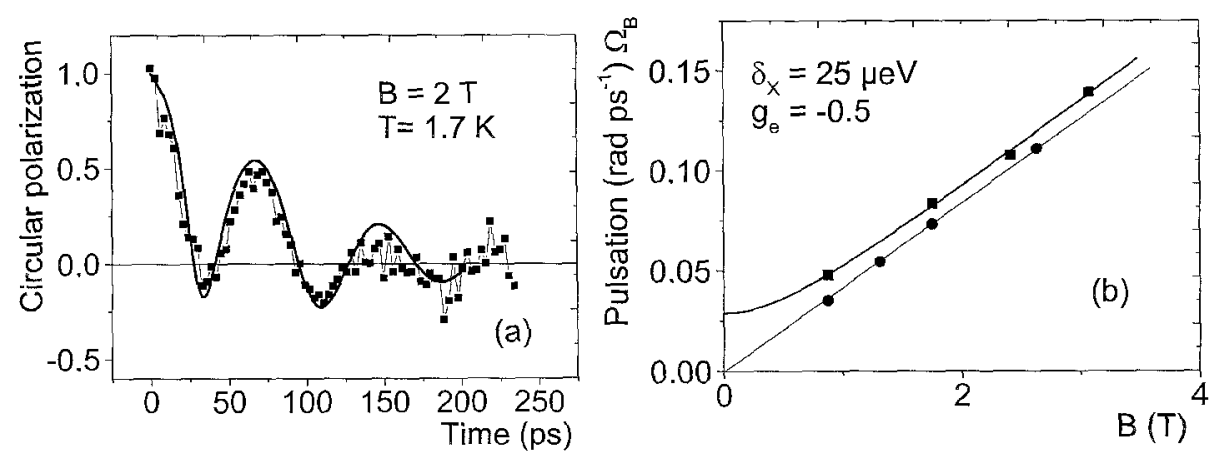

Fig. 12. $\mathrm{Ga}_{0.94} \mathrm{In}_{0.06}$ As/GaAs quantum well structure (well width $=4 \mathrm{~nm}$ ) in a transverse magnetic field $B_{x}=2 \mathrm{~T}$. (a) Intensity and polarization dynamics after a resonant $\sigma^{+}$excitation pulse; the fitting parameters are: $\tau_{\mathrm{h}}=60 \mathrm{ps}, \tau_{\mathrm{e}}=130 \mathrm{ps}, T_{s 1}=80 \mathrm{ps}$, $\tau_{\text {rad }}=30$ ps. (b) The beat period dependence on $B_{x}$ for resonant ( $(\mathbf{c})$ and non-resonant $(\bullet)$ excitation, from which the parameters $\delta_{X}=25 \pm 5 \mu \mathrm{eV}$ and $g_{\mathrm{e}, \perp}=-0.5 \pm 0.01$ are extracted with the appropriate fit (see the text).

that from the two types of experiments an accurate value of the exchange energy $\delta_{X}=\sqrt{\left(\hbar \Omega_{B}\right)^{2}+\left(\hbar \omega_{\mathrm{L}}\right)^{2}}$ can be measured (for instance, $\delta_{X}=130 \pm 15 \mu \mathrm{eV}$ for the quantum well of Fig. 10). Figure 12 illustrates a situation encountered in $\mathrm{Ga}_{0.94} \mathrm{In}_{0.06} \mathrm{As} / \mathrm{GaAs}$ quantum wells, where $g_{\mathrm{e}, \perp}$ is larger than the ones in GaAs QW and $\delta_{X}$ is smaller, leading to a stronger mixing between the $J=1$ and $J=2$ states. The fits are obtained adding the general damping terms to Eq. (18) as specified in Annex 2. We observe that, at early time delays, the $I^{+}$extinctions are more pronounced, and, when time increases, the polarization oscillations become symmetrical with respect to the zero polarization axis, due to the progressive hole spin relaxation.

Finally, exciton quantum beats, which can be observed during a few tens of picosecond following a polarized excitation pulses under magnetic field, are the manifestation of the collective exciton spin coherence. The latter is enhanced by confinement which increases the electron-hole exchange interaction and stabilizes the hole spin. Thus, two-dimensional excitons can keep the memory of the photon angular momentum which have generated them, on a much longer time than the phase memory of the excitation laser pulse.

These experiments have been extended recently to positively charged excitons, which behave like electron-spins under magnetic field [41], and microcavity polaritons where the effective $g$-factor can be modified with the cavity detuning $[42]$. 


\section{Exciton spin dynamics in semiconductor self-assembled quantum dots}

We turn now to new semiconductor structures which provide a three-dimensional confinement, the so-called quantum dots (QD). They consist in an island of small gap semiconductor, whose sizes are comparable or smaller than the three-dimensional exciton Bohr radius $(\sim 10 \mathrm{~nm})$, embedded in a matrix of wider gap material. In such QD, the discrete energy levels and the corresponding lack of energy dispersion lead to a predicted increase in the spin relaxation time compared to bulk or quantum well (QW) structures [43]. Here, we report on a direct time-resolved evidence of a spin relaxation quenching in InAs/GaAs QD.

The presented structure was grown by molecular beam epitaxy on a (001) GaAs substrate. It consists of 5 InAs QD planes embedded in a GaAs planar $\lambda$-microcavity designed so that the cavity mode $(\mathrm{FWHM} \approx 3 \mathrm{meV}$ ) can be tuned in the QD ground state energy range [44] (corresponding to the $X H$ excitonic transitions). The narrowing of the radiation pattern emitted by the microcavity allows us to collect the photoluminescence (PL) efficiently in spite of the small acceptance solid angle $\left(10^{-3}\right.$ steradians) of the up-conversion detection set-up we have used [11].

The sample is excited by $1.5 \mathrm{ps}$ linearly $\left(\sigma^{x}\right)$ or circularly $\left(\sigma^{+}\right)$polarized pulses generated by an optical parametric oscillator. The laser excitation energy coincides with both the cavity mode and the QD ground state energy. The time-resolved PL is then detected at the excitation energy by a two-color up-convertion set-up $[11,45]$.

The linear and the circular polarization degrees of the PL are defined as previously. Note that the linear polarization is taken along the axis parallel to the $[1,1,0]$ and $[1,-1,0]$ sample directions. The experiments have been performed at low excitation power $\left(\sim 7 \mathrm{~W} \mathrm{~cm}^{-2}\right)$ which corresponds to an average estimated density of photoexcited carriers less than one electron-hole pair per QD.

In the envelope function approximation, and keeping the growth direction $\mathrm{O} z$ as the quantization axis, the angular momentum of electron and holes states are still described by Bloch states as in the previous Sec. 2. In self-organized QD structures however, the symmetry is lowered with respect to the one of quantum wells, since the QD are usually shaped in the form of truncated-pyramids or lenses [46]. The QD are then no more invariant by the roto-inversion symmetry operation and the exchange interaction is no more isotropic [3].

Assuming QD with square bases ( $C_{2 v}$ symmetry), the anisotropic exchange interaction splits now the $| \pm 1\rangle$ radiative doublets into the two eigenstates labeled $|X\rangle=(|1\rangle+|-1\rangle) / \sqrt{2}$ and $|Y\rangle=(|1\rangle-|-1\rangle) / \mathrm{i} \sqrt{2}$, linearly polarized along the $[1,1,0]$ and $[1,-1,0]$ directions, respectively for QD with bases oriented along the [100] and [010] crystal directions as ours [47]. Continuous wave (cw) single dot spectroscopy experiments have clearly evidenced these two linearly polarized lines in self-organized InGaAs QD with an exchange splitting of $\hbar \omega \approx 150 \mu \mathrm{eV}$ [48]. 

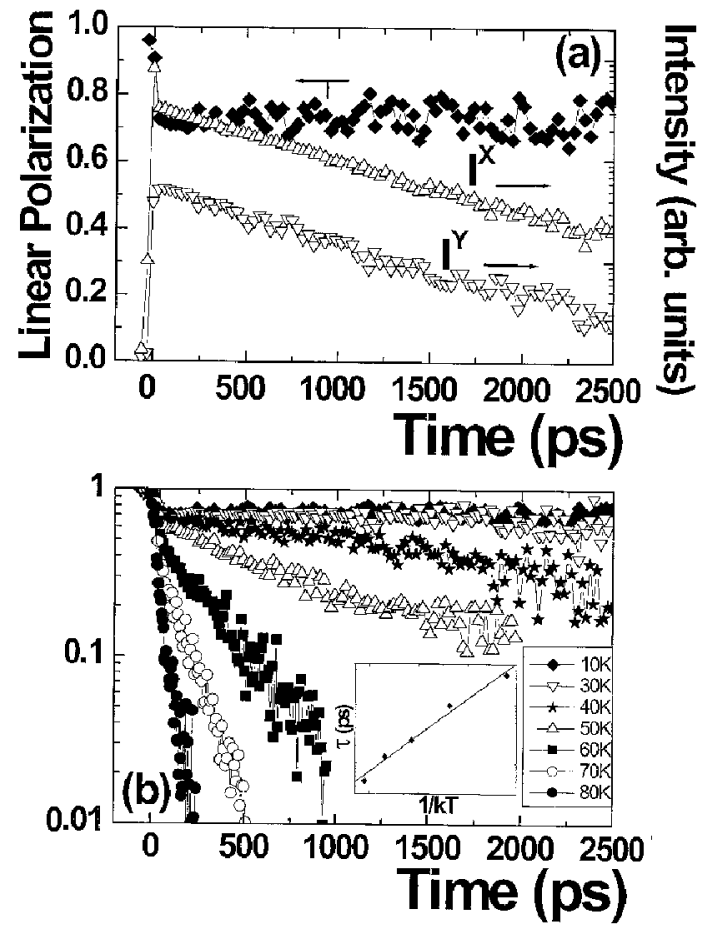

Fig. 13. (a) Time dependence of the PL components co-polarized $I^{x}$ and cross-polarized $I^{y}$ to the linearly polarized $\left(\sigma^{x}\right)$ excitation laser $(T=10 \mathrm{~K})$ and the corresponding linear polarization $P_{\text {lin }}$ (full $\diamond$ ) (the initial peak on the $I^{x}$ luminescence component intensity corresponds to backscattered laser light from the sample surface). (b) Temperature dependence of the linear polarization dynamics. Inset $-P_{\text {lin }}$ decay time as a function of $1 /\left(k_{\mathrm{B}} T\right)$

Figure 13a displays the time dependence of the PL components co-polarized $\left(I^{x}\right)$ and cross-polarized $\left(I^{y}\right)$ to the $\sigma^{x}$ polarized excitation laser and the corresponding linear polarization. The QD emission exhibits a strong linear polarization $\left(P_{\text {lin }} \approx 0.75\right)$ which remains strictly constant within our experimental accuracy during the exciton emission (i.e. over $\sim 2.5 \mathrm{~ns}$ ). This behavior differs strongly from the exciton linear polarization dynamics in bulk or type I QW structures, characterized by a linear polarization decay time of a few tens of picoseconds as seen in Sec. 2. In the latter structures, it has been demonstrated that any scattering mechanism (phonon, exchange interaction, electron and/or hole single particle spin relaxation) results in the destruction of the coherent superposition between the $|1\rangle$ and $|-1\rangle$ components of the linear exciton. The experimental observation of a QD exciton linear polarization which does not decay with time is the proof that neither the electron, nor the hole, nor the exciton spin relax on the exciton lifetime scale. 
Figure 13b presents the dependence of the exciton PL linear polarization dynamics upon the lattice temperature. A clear temporal decay of $P_{\text {lin }}$ is observed above $30 \mathrm{~K}$, the linear polarization decay time drops from $\sim 3500$ ps at $40 \mathrm{~K}$ down to $50 \mathrm{ps}$ at $80 \mathrm{~K}$ with an activation energy $E_{\mathrm{a}}=30 \pm 1 \mathrm{meV}$. This strong temperature dependence can be due either to any electron, hole, exciton spin-flip scattering or any spin-conserving scattering processes which break the coherent superposition of the linearly photogenerated excitons $|X\rangle$. To the best of our knowledge there is no available theory on the exciton spin-flip and its temperature dependence in QD. However one can speculate that the depolarization mechanism is due to hole scattering to higher QD excited states since the measured activation energy is close both to the energy splitting between the ground and first excited heavy-hole state and to the InAs LO phonon energy [49].

A circularly polarized excitation should lead to the observation of circular polarization quantum beats at the pulsation corresponding to the anisotropic exchange splitting. After about $15 \mathrm{ps}$, the time required for the QD PL signal to overcome the backscattered laser light, we do not observe any beat in this excitation configuration. This absence is interpreted as a consequence of the exchange splitting energy statistical fluctuations among the QD whose energy coincides with the cavity mode.

Under magnetic field $(B)$ applied along the growth direction, if the Zeeman splitting $\hbar \Omega_{B}=\left(g_{\mathrm{e}, \|}+g_{\mathrm{h}, \|}\right) \mu_{\mathrm{B}} B_{z}$ is much larger than the anisotropic exchange energy $\hbar \omega$, the QD exciton eigenstates are no more the $|X\rangle$ and $|Y\rangle$ linearly polarized states but the $|+1\rangle$ and $|-1\rangle$ circular ones. We thus expect to observe a

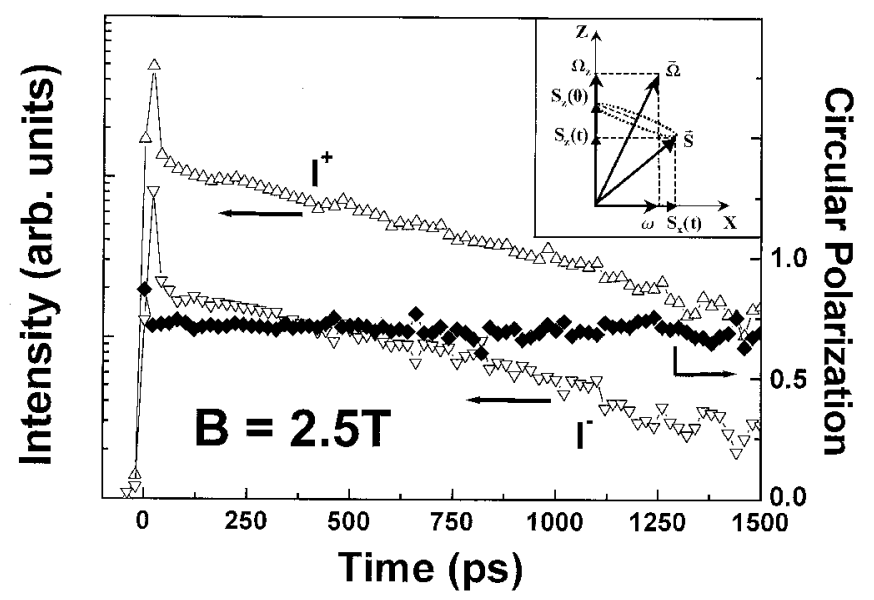

Fig. 14. Time dependence of the PL components co-polarized $I^{+}$and counter-polarized $I^{-}$to the $\sigma^{+}$-polarized excitation laser $(T=1.7 \mathrm{~K})$ and the corresponding circular polarization $P_{\text {circ }}($ full $\diamond)$. Inset: schematic representation of the exciton pseudo spin $\boldsymbol{S}(t)$ rotating around the vector $\Omega$ after $\sigma^{+}$polarized excitation (see the text). 
circularly polarized PL under $\sigma^{+}$-polarized excitation, as already measured in stationary spectroscopy $[46,48]$. This circular polarization is indeed observed (see Fig. 2, where $B=2.5 \mathrm{~T}$ ) and approaches $100 \%$ for $B=5 \mathrm{~T}$. Again, the striking feature is the absence of any polarization decay on the exciton emission scale which confirms that the QD exciton spin is totally frozen.

We also observe, under magnetic field, a clear conversion from optical orientation to alignment (i.e. detection of linearly polarized PL after excitation by $\sigma^{+}$-polarized pulses) and vice versa [46], but once more the linear or circular polarization remains temporally constant.

The circular or linear exciton polarization dynamics can be described in the framework of an exciton pseudospin with $S=1 / 2$ [46], as introduced in Sec. 2. Considering QD with square bases, the pseudospin Hamiltonian that takes into account the exchange and the Zeeman terms is simply equal to [46]

$$
H_{\mathrm{QD}}=\hbar\left(\omega \sigma_{x}+\Omega_{B} \sigma_{z}\right) / 2 .
$$

The schematic representation shown in the inset of Fig. 14 shows that the pseudo-spin rotates around the vector $\Omega\left(\omega, 0, \Omega_{B}\right)$ after a circularly polarized excitation characterized by an initial pseudo-spin $\boldsymbol{S}(0)$ parallel to the $O z$ axis. The projection of $\boldsymbol{S}(t)$ on the $O z$ axis yields the circular PL polarization which should oscillate as a function of time. We do not observe in Fig. 14 any beating structure in the PL circular polarization. As already discussed above, this absence comes from the exchange energy dispersion among the detected QD. We emphasize that this dispersion, which leads to the destruction of the PL beats, does not prevent the observation of circular polarization since the mean QD pseudospin projection on $O z$ is non-zero and tends to $1 / 2$ when $B$ increases.

Under linearly-polarized excitation, the initial pseudospin $\boldsymbol{S}(0)$ is parallel to the $O x$ axis. Increasing the magnetic field yields a reduction of the average value of the PL exciton linear polarization $P_{\operatorname{lin}}(t)=2 S_{x}(t)$. The observed magnetic field dependence of the PL circular and linear polarization displayed in Figs. 15a and $15 \mathrm{~b}$, respectively, confirms this interpretation.

Moreover we see in the inset of Fig. 14 that under a linearly (circularly)-polarized excitation, one expects to observe a non-zero circular $P_{\text {circ }}$ (linear $P_{\text {lin }}$ ) photoluminescence polarization. This conversion from optical orientation to alignment and vice versa, reported in Figs. 15c and 15d, was previously observed for excitons in type II QWs and in InAlAs/AlGaAs QD; it is a direct consequence of the anisotropic exciton exchange interaction [46, 50].

The magnetic field dependence of the linear and circular polarization dynamics allows the determination of the exciton fine structure [51]. Following Dzhioev et al., a quantitative description of these dependences can be given by $[46,50]$

$$
P_{\text {circ }} \cong P_{\text {circ }}^{0} \frac{\tilde{\Omega}_{B}^{2}}{\tilde{\omega}^{2}+\tilde{\Omega}_{B}^{2}} \quad(\mathrm{a}), \quad P_{\text {lin }} \cong P_{\operatorname{lin}}^{0} \frac{\tilde{\omega}^{2}}{\tilde{\omega}^{2}+\tilde{\Omega}_{B}^{2}} \quad \text { (b), }
$$




$$
P_{\text {circ }} \cong P_{\text {lin }}^{0} \frac{\tilde{\omega} \tilde{\Omega}_{B}}{\tilde{\omega}^{2}+\tilde{\Omega}_{B}^{2}} \quad(\mathrm{c}), \quad P_{\text {lin }} \cong P_{\text {circ }}^{0} \frac{\tilde{\omega} \tilde{\Omega}_{B}}{\tilde{\omega}^{2}+\tilde{\Omega}_{B}^{2}} \quad(\mathrm{~d}),
$$

which correspond, respectively, to the experiments presented in Figs. 15a-d. Here, $(\tilde{A})^{2}$ represents the statistical average of $A^{2}$ over the QD distribution $\left(A=\omega, \Omega_{B}\right)$. $P_{\text {circ }}^{0}$ and $P_{\text {lin }}^{0}$ are fitting parameters which denote, respectively, the circular or linear initial $(t=0)$ polarization. The four curves (solid lines) displayed in Fig. 15 are the best fits to the experimental data obtained using Eqs. (20a-d) with the following parameters: $P_{\text {circ }}^{0}=0.95, P_{\text {lin }}^{0}=0.75$, and $\left|\tilde{g}_{X}\right| / \tilde{\omega}=14.5 \mathrm{ps}\left(g_{X}=g_{\mathrm{e}, \|}+g_{\mathrm{h}, \|}\right)$. Bayer et al. have measured the exciton $g$-factor value $\left(\left|\tilde{g}_{X}\right| \sim 3\right)$ in InGaAs QD by single dot magneto-photoluminescence spectroscopy [48]. If we assume that this $\left|\tilde{g}_{X}\right|$ value is also relevant for our quantum dot, this leads to $\hbar \tilde{\omega}=135 \mu \mathrm{eV}$. We attribute the discrepancy between the fit parameter $P_{\mathrm{lin}}^{0}=0.75$ and the measured initial polarization $P_{\text {lin }}^{\text {laser }}=0.95$ to our very simple approach which ignores any orientation fluctuations from the $[1,1,0]$ and the $[1,-1,0]$ orientated eigenstates. These slight fluctuations may originate from statistical fluctuations of the QD shape and orientation [46].

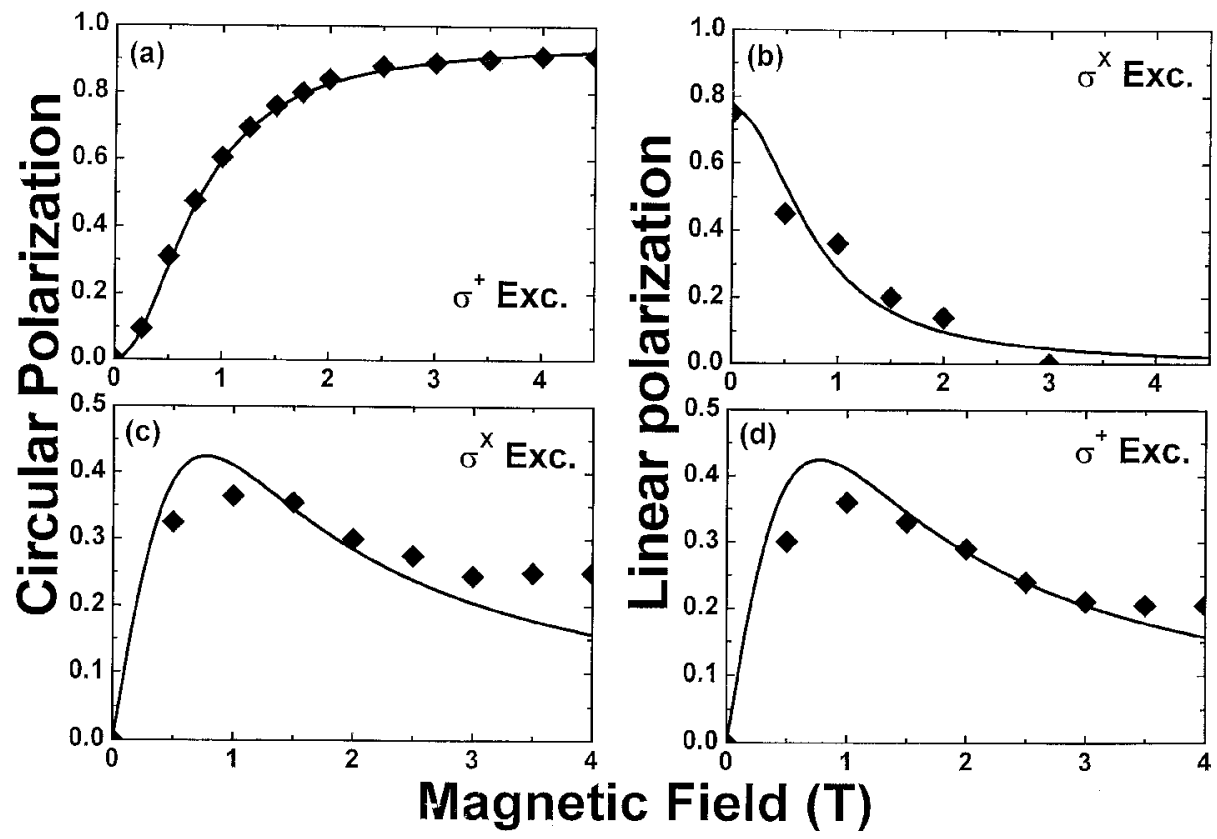

Fig. 15. Magnetic field dependence of the circular (linear) polarization under circularly polarized $\sigma^{+}[(\mathrm{a}),(\mathrm{d})]$ and linearly polarized $\sigma^{x}$ excitation [(b),(c)] ( $\left.T=1.7 \mathrm{~K}\right)$. The displayed polarization values are the one measured at any time delay after the pulsed excitation, since we do not observe any polarization decay. The solid lines are the best fits obtained with Eqs. (1a-d), $\left|\tilde{g}_{X}\right| / \tilde{\omega}=14.5$ ps (see the text). 
In conclusion, we have studied the linear and circular PL polarization dynamics in self-organized QD under strictly resonant excitation. We never observe at low temperature any measurable temporal decay of the linear or circular luminescence polarization regardless of the excitation polarization and the magnetic field value. This evidences a complete spin relaxation quenching in these zero-dimensional structures. These results contrast with the clear spin relaxation previously observed in non-resonant excitation conditions [52]. The main difference between these two kinds of experiments relies on the higher energy carrier state occupation, including barrier, wetting layer, and QD excited states which may induce the spin flip of the QD ground state by Coulomb exchange. Spin dynamics under magnetic field is somehow obscured by the inherent size dispersion encountered in such systems. It is thus highly desirable to perform experiments on single QD, in order to observe a Hamiltonian spin evolution, as recently observed in CdSe microcrystals [53].

\section{Conclusion}

We have shown in this brief review how to manipulate spin with optical pulses, and the basics of spin dynamics under magnetic field in various semiconductor structures. Other systems with high potential interest with respect to carrier spin manipulations have not been described here, as $[1,1,0]$ oriented GaAs/AlGaAs quantum wells where the D'Yakonov and Perel mechanism is substantially suppressed [54], doped semiconductor structures [55], semimagnetic structures (including e.g. CdMnSe [56], or GaMnAs materials [57]), and hybrid ferromagnetic metal/semiconductor devices, designed to achieve electrical spin injection and transport [58]. On the other hand, the observation of a quenched spin relaxation in self-assembled quantum dots structures brings clear experimental support to proposals [59] using electron spins for quantum information encoding and processing in a solid state system.

\section{Annex 1}

It can be inferred from Ref. [60] that the macroscopic polarization of the microcavity following a single $\sigma^{+}$(or $\sigma^{-}$) optical pulse can be expressed in the frequency domain by

$$
\begin{aligned}
& \boldsymbol{P}_{ \pm}(\hbar \omega) \approx N\left|\mu_{\oslash, 1}\right|^{2} \frac{T E_{X H}}{2 \pi\left(E_{U B}-E_{L B}\right)} \\
& \times\left(\frac{1}{\hbar \omega-E_{L B}+\mathrm{i} \Gamma_{L B} / 2}-\frac{1}{\hbar \omega-E_{U B}+\mathrm{i}} \Gamma_{U B} / 2\right) \mathcal{E}\left(\hbar \omega-\hbar \omega_{0}\right) \boldsymbol{e}_{ \pm}
\end{aligned}
$$

where $\mu_{\oslash, 1}$ is the quantum well dipolar matrix element between the ground state and $J_{z}= \pm 1$ angular momentum of heavy-hole excitons, $N$ is the number of 
quantum wells located at the antinodes of the microcavity (e.g. $N=4$ here), $E_{X H}$ is the bare heavy-hole exciton energy, $T$ is the transmission coefficient of the Bragg mirrors, $E_{L B(U B)}$ are the two polariton states energies, $\Gamma_{L B(U B)}$ are the corresponding polariton homogeneous broadening. Finally, $\mathcal{E}\left(\hbar \omega-\hbar \omega_{0}\right)$ is the Fourier transform of the incoming electric field envelope function, $\omega_{0}$ - its central frequency and $\boldsymbol{e}_{ \pm}=\left(\boldsymbol{e}_{X} \pm \mathrm{i} \boldsymbol{e}_{Y}\right) / \sqrt{2}$. The temporal polarization function is given by

$$
\boldsymbol{P}(t)=\frac{1}{2 \pi} \int \boldsymbol{P}(\hbar \omega) \exp (-\mathrm{i} \omega t) \mathrm{d} \omega .
$$

When we apply an external picosecond optical pulse, resonant with the lower polariton branch, with a spectral width broader than the polariton damping, but much smaller than the Rabi splitting (which implies that the condition $\Gamma_{\mathrm{e}}, \Gamma_{\mathrm{c}} \ll \hbar \Omega_{\mathrm{R}}$ is fulfilled), the temporal response writes

$$
\begin{aligned}
& \boldsymbol{P}_{ \pm}(t) \approx P(0) \exp \left(-\frac{\Gamma_{L B}}{2 \hbar} t\right) \exp \left(-\mathrm{i} \frac{E_{L B}}{\hbar} t\right) \theta(t) \boldsymbol{e}_{ \pm}, \\
& P(0)=-\mathrm{i} N\left|D_{\oslash, 1}\right|^{2} \frac{T E_{\text {ex }}}{2 \pi \hbar \Omega} \mathcal{E}(0),
\end{aligned}
$$

where $\theta(t)$ is the Heaviside step function.

We can now proceed to the coherent control experiment. The total microcavity polarization following a $\left(\sigma^{+}, \sigma^{-}\right)$excitation sequence writes

$$
\boldsymbol{P}(t)=\boldsymbol{P}_{+}(t)+\boldsymbol{P}_{-}(t-\tau)
$$

where $\tau=t_{1}+t_{2}$ is the temporal separation between the two pulses. For $t>\tau$, assuming equal intensity pulses, $\boldsymbol{P}(t)$ writes in the $\boldsymbol{e}_{X}, \boldsymbol{e}_{Y}$ basis

$$
\begin{aligned}
& \boldsymbol{P}(t)=P(0) \exp \left(-\frac{\Gamma_{L B}}{2 \hbar} t\right) \exp \left(-\mathrm{i} \frac{E_{L B}}{\hbar} t\right) \\
& \times\left[\left(\frac{1+\exp \left(\frac{\Gamma_{L B} / 2+\mathrm{i} E_{L B}}{\hbar} \tau\right)}{\sqrt{2}}\right) \boldsymbol{e}_{x}+\left(\frac{1-\exp \left(\frac{\Gamma_{L B} / 2+\mathrm{i} E_{L B}}{\hbar} \tau\right)}{\sqrt{2}}\right) \mathrm{i} e_{y}\right] .
\end{aligned}
$$

The total $L B$ polariton population $N(t)$ is given by

$$
N(t)=\left|\boldsymbol{P}_{+}(0)\right|^{2} \exp \left(-\frac{t}{T_{1}}\right) \theta(t)+\left|\boldsymbol{P}_{-}(0)\right|^{2} \exp \left(-\frac{t-\tau}{T_{1}}\right) \theta(t-\tau)
$$

and the total emission intensity in the observation direction is proportional to $N(t)$. The linear polarization of the SE, detected in a non specular direction, writes

$$
P^{l}(t)=\frac{I_{\mathrm{coh}}^{X}(t)+I_{\mathrm{inc}}^{X}(t)-\left[I_{\mathrm{coh}}^{Y}(t)+I_{\mathrm{inc}}^{Y}(t)\right]}{N(t)},
$$

where $I_{\mathrm{coh}}^{X(Y)}$ are the intensities of the two linearly polarized components of the RRS in the detected direction, and $I_{\text {inc }}^{X(Y)}$ those of the incoherent polaritons emission. 
Just after the second pulse $(t=\tau+0)$, the incoherent contribution to the signal is negligible, provided that $t \tilde{<} T_{2}\left(=2 \hbar / \Gamma_{L B}\right)$ and the emission linear polarization obtained as stated in Eq. (14).

\section{Annex 2}

The phenomenological relaxation terms in the general case take into account the electron and hole single particle spin relaxations (characteristic times $\tau_{\mathrm{e}}, \tau_{\mathrm{h}}$ ), the exciton spin relaxation, and the radiative exciton recombination (time $\tau_{\text {rad }}$ ). They can be written as

$$
\begin{aligned}
& \frac{\mathrm{d} s_{\perp}}{\mathrm{d} t}=-\frac{s_{\perp}}{2}\left(\frac{1}{\tau_{\mathrm{e}}}+\frac{1}{2 T_{s 1}}+\frac{1}{\tau_{\mathrm{rad}}}\right) \\
& \frac{\mathrm{d} s_{z}}{\mathrm{~d} t}=-\frac{s_{z}}{\tau_{\mathrm{e}}}-\frac{1}{2}\left(s_{z}-\frac{j_{z}}{3}\right)\left(\frac{1}{T_{s 1}}+\frac{1}{\tau_{\mathrm{rad}}}\right), \\
& \frac{\mathrm{d} j_{z} / 3}{\mathrm{~d} t}=-\frac{j_{z} / 3}{\tau_{\mathrm{h}}}-\frac{1}{2}\left(\frac{j_{z}}{3}-s_{z}\right)\left(\frac{1}{T_{s 1}}+\frac{1}{\tau_{\mathrm{rad}}}\right), \\
& \frac{\mathrm{d} \boldsymbol{Q}_{\perp}}{\mathrm{d} t}=-\frac{\boldsymbol{Q}_{\perp}}{2}\left(\frac{1}{\tau_{\mathrm{e}}}+\frac{1}{2 T_{s 1}}+\frac{1}{\tau_{\mathrm{rad}}}\right)-\frac{\boldsymbol{Q}_{\perp}}{\tau_{\mathrm{h}}}, \\
& \frac{\mathrm{d} Q_{z}}{\mathrm{~d} t}=-\frac{1}{2 \tau_{\mathrm{rad}}}\left(Q_{z}-\frac{N}{2}\right)-Q_{z}\left(\frac{1}{\tau_{\mathrm{h}}}+\frac{1}{\tau_{\mathrm{e}}}\right) \\
& \frac{\mathrm{d} N}{\mathrm{~d} t}=-\frac{1}{2 \tau_{\mathrm{rad}}}\left(\frac{N}{2}-Q_{z}\right),
\end{aligned}
$$

where $\boldsymbol{s}_{\perp}=\left(s_{x}, s_{y}\right), \boldsymbol{Q}_{\perp}=\left(Q_{x}, Q_{y}\right)$, and $N$ is the total exciton population.

\section{References}

[1] C. Monroe, D. Meekhof, B. King, W. Itano, W. Wineland, Phys. Rev. Lett. 75, 4714 (1995).

[2] L.C. Andreani, in: Confined Electrons and Photons, Eds. E. Burstein, C. Weisbuch, Plenum Press, New York, NATO ASI Series B: Physics 340, 57 (1995).

[3] E. Blackwood, M.J. Snelling, R.T. Harley, S.R. Andrews, C.T.B. Foxon, Phys. Rev. B 50, 14246 (1994).

[4] M.Z. Maialle, E.A. de Andrada e Silva, L.J. Sham, Phys. Rev. B 47, 15776 (1993).

[5] see e.g. Optical Orientation, Eds. F. Meier, B. Zakharchenya, in series Modern Problems in Condensed Matter Science, Vol. 8, North-Holland, Amsterdam 1984.

[6] M. Oestreich, D. Hägele, J. Hübner, W.W. Rühle, Phys. Status Solidi A 178, 27 (2000). 
[7] A. Vinattieri, J. Shah, T.C. Damen, D.S. Kim, L.N. Pfeiffer, M.Z. Maialle, L.J. Sham, Phys. Rev. B 50, 10868 (1994).

[8] P. Le Jeune, X. Marie, T. Amand, F. Romstad, F. Perez, J. Barrau, M. Brousseau, Phys. Rev. B 58, 4853 (1998).

[9] A.P. Heberle, J.J. Baumberg, K. Köhler, Phys. Rev. Lett. 75, 2598 (1995).

[10] D.S. Yee, K.J. Yee, D.S. Kim, in: Quatum Electronics and Laser Science Conf., OSA Technical Digest, Optical Society of America, Washington (DC) 1999, p. 105.

[11] X. Marie, P. Le Jeune, T. Amand, M. Brousseau, J. Barrau, M. Paillard, Phys. Rev. Lett. 79, 3222 (1997).

[12] M. Woerner, J. Shah, in: Ultrafast Phenomena XI, Eds. T. Elsaesser, J.G. Jujimoto, D.A. Wiersma, W. Zinth, in Springer Series in Chemical Physics, Vol. 63, Springer, Berlin 1998, p. 227; M. Woerner, J. Shah, Phys. Rev. Lett. 81, 4208 (1998)

[13] X. Marie, T. Amand, P. Le Jeune, M. Brousseau, J. Barrau, in: Coherent Control in Atoms, Molecules, and Semiconductors, Eds. W. Pötz, W.A. Schroeder, Kluwer Academic Publishers, Dordrecht 1999, p. 87.

[14] P. Renucci, M. Paillard, X. Marie, T. Amand, J. Barrau, C. Ciuti, Phys. Status Solidi A 178, 373 (2000); see also J. Erland, V.G. Lyssenko, J.M. Hvam, Phys. Rev. B 63, 15317 (2001).

[15] J. Kuhl, E.J. Mayer, G. Smith, R. Eccleston, D. Bennhardt, P. Thomas, K. Bott, O. Heller, in: Coherent Optical Interactions in Semiconductors, Ed. R.T. Phillips, Plenum Press, New York, NATO ASI Series B: Physics 330, 1 (1994).

[16] S. Haacke, S. Schaer, B. Deveaud, V. Savona, in: Ultrafast Phenomena in Semiconductors III, Ed. Kong Thon Tsen, Proc. SPIE 3624, 33 (1999).

[17] V. Savona, R. Zimmermann, Phys. Rev. B 60, 4928 (1999).

[18] C. Weisbuch, M. Nishioka, A. Ishikawa, Y. Arakawa, Phys. Rev. Lett. 69, 3314 (1992).

[19] A.I. Tartakovskii, V.D. Kulakovskii, D.N. Krizhanovskii, M.S. Skolnick, V.N. Astranov, A. Armitage, J.S. Roberts, Phys. Rev. B 60, R11293 (1999).

[20] C. Ciuti, Ph.D. Thesis, Swiss Federal Institute of Technology, Lausanne 2001.

[21] P.G. Savvidis, J.J. Baumberg, R.M. Stevenson, M.S. Skolnick, D.M. Whittaker, J.S. Roberts, Phys. Rev. Lett. 84, 1547 (2000).

[22] C. Ciuti, P. Schwendimann, B. Deveaud, A. Quattropani, Phys. Rev. B 63, R41303 (1901).

[23] P. Renucci, X. Marie, T. Amand, M. Paillard, P. Senellart, J. Bloch, in: Proc. 25th Int. Conf. Phys. Semicond., Osaka 2000, Eds. N. Miura, T. Ando, in series Springer Proceedings in Physics, Vol. 87, Springer, Berlin 2001, p. 653.

[24] X. Marie, P. Renucci, S. Dubourg, T. Amand, P. Le Jeune, J. Barrau, J. Bloch, R. Planel, Phys. Rev. B 59, R2494 (1999).

[25] T. Amand, X. Marie, P. Renucci, M. Paillard, J. Barrau, in: Ultrafast Phenomena in Semiconductors III, Ed. Kong Thon Tsen, Proc. SPIE 3624, 92 (1999).

[26] T.B. Norris, I.K. Rhee, D.S. Citrin, M. Nishioka, Y. Arakawa, Nuovo Cimento 17, 1295 (1995). 
[27] V. Savona, C. Piermarocchi, A. Quattropani, F. Tassone, P. Schwendimann, Phys. Rev. Lett. 78, 4470 (1997).

[28] T. Freixanet, B. Sermage, J. Bloch, J.Y. Marzin, R. Planel, Phys. Rev. B 60, R8509 (1999).

[29] H. Wang, J. Shah, T.C. Damen, W.Y. Jan, J.E. Cunningham, M. Hong, J.P. Mannaerts, Phys. Rev. B 51, 14713 (1995).

[30] L. Schultheis, A. Honold, J. Kuhl, K. Köhler, C.W. Tu, Phys. Rev. B 34, 9027 (1986); K. Leo, E.O. Göbel, T.C. Damen, J. Shah, S. Schmitt-Rink, W. Schäfer, J.F. Müller, K. Köhler, P. Ganser, Phys. Rev. B 44, 5726 (1991).

[31] V. Savona, C. Piermarocchi, Phys. Status Solidi A 164, 45 (1997).

[32] R.E. Worsley, N.J. Traynor, T. Grewatt, R.T. Harley, Phys. Rev. Lett. 76, 3224 (1996).

[33] I.V. Mashkov, C. Gourdon, P. Lavallard, D. Yu. Roditchev, Phys. Rev. B 55, 13761 (1997).

[34] S. Bar-Ad, I. Bar-Joseph, Phys. Rev. Lett. 66, 2491 (1991).

[35] T. Amand, X. Marie, M. Brousseau, P. Le Jeune, D. Robart, J. Barrau, R. Planel, Phys. Rev. Lett. 78, 1355 (1997).

[36] M. D'Yakonov, X. Marie, T. Amand, P. Le Jeune, D. Robart, M. Brousseau, J. Barrau, Phys. Rev. B 56, 10412 (1997).

[37] X. Marie, T. Amand, P. Le Jeune, M. Paillard, P. Renucci, L.E. Golub, V.M. Dymnikov, E.L. Ivchenko, Phys. Rev. B 60, 5811 (1999).

[38] A.P. Heberle, W.W. Rühle, K. Ploog, Phys. Rev. Lett. 72, 3887 (1994).

[39] R.M. Hannak, M. Oestreich, A.P. Heberle, W.W. Rühle, K. Köhler, Solid State Commun. 93, 313 (1995).

[40] B. Baylac, X. Marie, T. Amand, M. Brousseau, J. Barrau, Y. Shekun, Surf. Sci. 326, 161 (1995).

[41] E. Vanelle, M. Paillard, X. Marie, T. Amand, P. Gilliot, D. Brinkmann, R. Lévy, J. Cibert, S. Tatarenko, Phys. Rev. B 60, 15542 (2000).

[42] P. Renucci, X. Marie, T. Amand, M. Paillard, E. Vanelle, in: Proc. 25th Int. Conf. Phys. Semicond., Osaka 2000, Eds. N. Miura, T. Ando, in series Springer Proceedings in Physics, Vol. 87, Berlin 2001, p. 661.

[43] A.V. Khaetskii, Y. Nazarov, Phys. Rev. B 61, 12639 (2000); A.V. Khaetskii, Y. Nazarov, Physica E 6, 470 (2000).

[44] J.M. Gérard, B. Sermage, B. Gayral, B. Legrand, E. Costard, V. Thierry-Mieg, Phys. Rev. Lett. 81, 1110 (1998).

[45] M. Paillard, X. Marie, E. Vanelle, T. Amand, V.K. Kalevich, A.R. Kovsh, A.E. Zhukov, V.M. Ustinov, Appl. Phys. Lett. 76, 76 (2000).

[46] E.L. Ivchenko, Pure Appl. Chem. 67, 463 (1995); R.I. Dzhioev, B.P. Zakharchenya, E.L. Ivchenko, V.L. Korenev, Yu.G. Kusraev, N.N. Ledensov, V.M. Ustinov, A.E. Zhukov, A.E. Tsatsul'nikov, Phys. Solid State 40, 790 (1998).

[47] S. Hameau, Y. Guldner, O. Verzelen, R. Ferreira, G. Bastard, J. Zeman, A. Lemaitre, J.M. Gérard, Phys. Rev. Lett. 83, 4152 (1999). 
[48] M. Bayer, A. Kuther, A. Forchel, A. Gorbunov, V.B. Timofeev, F. Schäfer, J.P. Reithmaier, T.L. Reinecke, S.N. Walck, Phys. Rev. Lett. 82, 1748 (1999).

[49] S. Sauvage, P. Boucaud, T. Brunhes, A. Lemaitre, J.M. Gérard, Phys. Rev. B 60, 15589 (1999).

[50] R.I. Dzhioev, H.M. Gibbs, E.L. Ivchenko, G. Khitrova, V.L. Korenev, M.N. Tkachukand, B.P. Zakharchenya, Phys. Rev. B 56, 13405 (1997); C. Gourdon, P. Lavalard, Phys. Rev. B 46, 4644 (1992).

[51] M. Paillard, X. Marie, P. Renucci, T. Amand, A. Jbeli, J.M. Gérard, Phys. Rev. Lett. 86, 1634 (2001).

[52] H. Gotoh, H. Ando, H. Kamada, A. Chavez-Pirson, J. Temmyo, Appl. Phys. Lett. 72, 1341 (1998).

[53] T. Flissikowski, A. Hundt, M. Lowisch, M. Rabe, F. Henneberger, Phys. Rev. Lett. 86, $3172(2001)$.

[54] Y. Ohno, R. Terauchi, T. Adachi, F. Matsukura, H. Ohno, Phys. Rev. Lett. 83, 4196 (1999).

[55] J.M. Kikkawa, D.D. Awschalom, Phys. Rev. Lett. 80, 4313 (1998).

[56] S.A. Crooker, J.J. Baumberg, F. Flack, N. Samarth, D.D. Awschalom, Phys. Rev. Lett. 77, 2814 (1996).

[57] H. Ohno, A. Shen, F. Matsukura, A. Oiwa, A. Endo, S. Katsumoto, Y. Iye, Appl. Phys. Lett. 69, 363 (1996).

[58] D. Hägele, M. Oestreich, W.W. Rühle, N. Nestle, K. Eberl, Appl. Phys. Lett. 73, 1580 (1998).

[59] G. Burkard, D. Loss, D.P. Di Vicenzo, Phys. Rev. B 59, 2070 (1999); G. Burkard, G. Seelig, D. Loss, Phys. Rev. B 62, 2581 (2000).

[60] D.S. Citrin, T.B. Norris, IEEE J. Sel. Topics Quantum Electron. 2, 401 (1996). 